\title{
Sortilin Fragments Deposit at Senile Plaques in Human Cerebrum
}

\author{
Xia Hu ${ }^{1}$, Zhao-Lan $\mathrm{Hu}^{1}$, Zheng $\mathrm{Li}^{2}$, Chun-Sheng Ruan ${ }^{3}$, Wen-Ying Qiu ${ }^{4}$, Aihua Pan ${ }^{1}$, \\ Chang-Qi Li ${ }^{1}$, Yan Cai ${ }^{1}$, Lu Shen ${ }^{5,6}$, Yaping Chu ${ }^{7}$, Bei-Sha Tang ${ }^{5,6}$, Huaibin Cai ${ }^{8}$, \\ Xin-Fu Zhou ${ }^{3}$, Chao Ma ${ }^{4}$ and Xiao-Xin Yan ${ }^{1,6 *}$ \\ ${ }^{1}$ Department of Anatomy and Neurobiology, Central South University School of Basic Medical Science, Changsha, China, \\ ${ }^{2}$ Cancer Research Institute, Central South University, Changsha, China, ${ }^{3}$ School of Pharmacy and Medical Sciences, \\ Sansom Institute, Division of Health Sciences, University of South Australia, Adelaide, SA, Australia, ${ }^{4}$ Department of Human \\ Anatomy, Histology and Embryology, Institute of Basic Medical Sciences, Neuroscience Center, Chinese Academy of Medical \\ Sciences, School of Basic Medicine, Peking Union Medical College, Beijing, China, ${ }^{5}$ Department of Neurology, Xiangya \\ Hospital, Central South University, Changsha, China, ${ }^{6}$ Key Laboratory of Hunan Province in Neurodegenerative Disorders, \\ Xiangya Hospital, Central South University, Changsha, China, 'Department of Neurological Sciences, Rush University \\ Medical Center, Chicago, IL, United States, ${ }^{8}$ Laboratory of Neurogenetics, National Institute on Aging, National Institutes \\ of Health, Bethesda, MD, United States
}

OPEN ACCESS

Edited by:

Alberto Munoz,

Complutense University of Madrid,

Spain

Reviewed by:

Giovanna Cenini,

University of Bonn, Germany

Fan Liao,

AbbVie Foundational Neuroscience Center, United States

*Correspondence: Xiao-Xin Yan yanxiaoxin@csu.edu.cn

Received: 08 April 2017 Accepted: 11 May 2017 Published: 07 June 2017

Citation: Hu X, Hu Z-L, Li Z, Ruan C-S, Qiu W-Y, Pan A, Li C-Q, Cai Y, Shen L, Chu Y, Tang B-S, Cai $H$,

Zhou X-F, Ma C and Yan X-X (2017) Sortilin Fragments Deposit at Senile Plaques in Human Cerebrum.

Front. Neuroanat. 11:45. doi: 10.3389/fnana.2017.00045
Genetic variations in the vacuolar protein sorting 10 protein (Vps10p) family have been linked to Alzheimer's disease (AD). Here we demonstrate deposition of fragments from the Vps10p member sortilin at senile plaques (SPs) in aged and AD human cerebrum. Sortilin changes were characterized in postmortem brains with antibodies against the extracellular and intracellular C-terminal domains. The two antibodies exhibited identical labeling in normal human cerebrum, occurring in the somata and dendrites of cortical and hippocampal neurons. The C-terminal antibody also marked extracellular lesions in some aged and all $A D$ cases, appearing as isolated fibrils, mini-plaques, densepacking or circular mature-looking plaques. Sortilin and $\beta$-amyloid $(A \beta)$ deposition were correlated overtly in a region/lamina- and case-dependent manner as analyzed in the temporal lobe structures, with co-localized immunofluorescence seen at individual SPs. However, sortilin deposition rarely occurred around the pia, at vascular wall or in areas with typical diffuse $A \beta$ deposition, with the labeling not enhanced by section pretreatment with heating or formic acid. Levels of a major sortilin fragment $\sim 15 \mathrm{kDa}$, predicted to derive from the C-terminal region, were dramatically elevated in $A D$ relative to control cortical lysates. Thus, sortilin fragments are a prominent constituent of the extracellularly deposited protein products at SPs in human cerebrum.

Keywords: Alzheimer's disease, amyloid deposition, neuritic plaques, synaptic pathology, Vps10p family proteins

\section{INTRODUCTION}

Senile plaques (SPs) were first described by Blocq and Marinesco in examination of silver stained brain samples from elderly epileptic patients (Critchley, 1929). The lesion was observed subsequently in the brains of elderly with and without dementia by other pioneer neuroscientists (e.g., Redlich, Alzheimer, Persini, Oppenheim, Fisher, Cajal), and it was Simvhowisz who named the pathology as "senile plaques" (Critchley, 1929; García-Marín et al., 2007; Ohry and Buda, 2015). Based on the silver preparation, it was also known by that time that SPs contained swollen neurites and some amorphous material-the former was named as dystrophic neurites (DNs) while the 
entire lesion as neuritic plaques. The term "amyloid plaques" was coined later by Divry who found Congo red stain of the amorphous material (Oŭfa, 1973). Electron microscopic studies and evidence from immunolabeling of presynaptic and neurotransmitter markers (e.g., synaptophysin (SYN), choline acetyltransferase, tyrosine hydroxylase, glutamate decarboxylase and vesicular glutamate transporters) in plaque-associated DNs suggest that they appear to be largely abnormal axons including presynaptic terminals (Luse and Smith, 1964; Gonatas et al., 1967; Struble et al., 1982, 1987; Walker et al., 1985; Masliah et al., 1991; Ferrer et al., 1998; Cai et al., 2010; Yan et al., 2014; Sadleir et al., 2016).

By the mid-1980s, $\beta$-amyloid peptides $(\mathrm{A} \beta)$ were identified from amyloid vasculature and parenchymal plaques in the human brain, marking a milestone in the history of research on cerebral $\beta$-amyloidosis relative to Alzheimer's disease ( $\mathrm{AD}$; Glenner and Wong, 1984; Masters et al., 1985). Within a few years antibodies to $A \beta$ became routine tools to stain SPs for definitive diagnosis of $\mathrm{AD}$. Site-specific deposition of $\mathrm{A} \beta$ at cerebral vasculature, meninge and diffuse plaques in the gray and white matter (WM) was also confirmed (Allsop et al., 1986; Jellinger and Bancher, 1988; Yamaguchi et al., 1988; Braak and Braak, 1991; Braak et al., 2006). The discovery of A $\beta$ as the key components of cerebral $\beta$-amyloidosis has since led to many other breakthroughs in the $\mathrm{AD}$ research field and beyond, as partially listed: (1) characterization of the amyloidogenic proteins, i.e., $\beta$-amyloid precursor protein (APP), $\beta$-secretase- 1 (BACE1) and $\gamma$-secretase complex (Robakis et al., 1987; Wolfe and Haass, 2001; Vassar et al., 2009); (2) establishment of the genetic link of APP and presenilin mutations to familial AD (Shea et al., 2016); (3) engineering of transgenic animal models of AD (Hsiao et al., 1996; Borchelt et al., 1997; Oddo et al., 2003; Oakley et al., 2006); (4) development of cerebrospinal fluid (CSF) biomarkers and $\mathrm{A} \beta$ imaging techniques for antemortem diagnosis of AD (Andreasen et al., 2001; Mathis et al., 2002; Herholz and Ebmeier, 2011); and (5) conceptualization of the anti-A $\beta$ therapy that has advanced from bench to bedside testing (Aisen, 2005; Yan et al., 2014; Karran and De Strooper, 2016). Notably, some blood proteins, heavy metals and lipoproteins accumulate around amyloid plaques (Coria et al., 1988; Rogers et al., 1992; Schwarzman et al., 1994; Eriksson et al., 1995; Kida et al., 1995; Watson et al., 1997; Burns et al., 2003; Wu et al., 2004; Garai et al., 2014; Cristóvão et al., 2016). Exploring additional plaque constituents, if any, may also help advance the understanding of plaque pathogenesis in the brain.

Variations in the vacuolar protein sorting 10 protein (Vps10p) family genes are recently shown to affect the risk of developing AD (Westergaard et al., 2004; Hermey, 2009). Variants in several loci of the sortilin-related receptor L1 gene (SORL1, also known as SORLA, SORLA1 or LR11) can increase the risk of $\mathrm{AD}$ (Rogaeva et al., 2007; Pottier et al., 2012; Wen et al., 2013; Felsky et al., 2014; Louwersheimer et al., 2015; Verheijen et al., 2016). Variants of single nucleotide polymorphisms (SNPs) of the sortilin related Vps10p domain containing receptor 1 (SORCS1) may also relate to AD (Reitz et al., 2011). The SNP rs17646665 of the sortilin gene (SORT1) is linked to a reduced risk of $\mathrm{AD}$ (Andersson et al., 2016). The Vps10p proteins belong to type
I transmembrane proteins, which might subject to proteolytic processing (Avci and Lemberg, 2015). The gene-association data obtained from human populations raise an intriguing issue as to whether the expression of some proteins from this family, including their potential fragment products, might be altered relative to $\mathrm{AD}$-type neuropathology.

With the setup of human brain banks, a preliminary batch of postmortem brains from Chinese donors became available for aging and AD-related studies (Zhu et al., 2015; Griffith et al., 2016). Using this resource, we conducted a pilot survey on the expression of the Vps10p members in the brain with commercial antibodies, and noticed that a sortilin C-terminal antibody visualized extracellular lesions appearing as amyloid plaques. Experiments were then carried out to verify this sortilin pathology, to profile its relationship with $A \beta$ deposition and to identify the candidate peptide component seen deposited in the plaques.

\section{MATERIALS AND METHODS}

\section{Human and Rodent Brain Samples}

Postmortem human brains were banked through the willed body donation programs, which existed with government (municipal police department and office of the Red Cross Society of China) and university approval to provide cadavers for teaching anatomy to medical students. Efforts are being taken to develop this platform for human brain banking as a part of the initiatives supporting China Brain Project (Yan et al., 2015; Poo et al., 2016). A subgroup of the elderly cases was recorded being dementia at the time of hospitalization for the care of terminal illnesses (Supplementary Table S1). Brain samples were assessed for $\mathrm{AD}$ type-neuropathology using sections from temporal, parietal, frontal and occipital lobes, with the extent of pathology (if present) scored according to Braak's staging and the NIH guideline (Jellinger and Bancher, 1988; Braak et al., 2006; Montine et al., 2012). Correlated anatomical and biochemical studies were carried out using samples from three groups designated according to age and AD-type neuropathology: (1) Mid-age cases $(n=9)$ died of non-neurological diseases and free of $\mathrm{A} \beta /$ tau pathology in the cerebrum; (2) Aged cases $(n=9)$ with a history of dementia but Braak's score of neurofibrillary tangle $\geq \mathrm{IV}$ and the $\mathrm{ABC}$ amyloid score $\geq \mathrm{B}$, defined as $\mathrm{AD}$ group, and (3) Aged control group $(n=9)$ with no $A \beta /$ tau pathology observed in the brain. It should be noted that, for comparative pathological analyses, additional aged cases with cerebral amyloidosis were included (Supplementary Table S1). Through this latter approach, brain samples/cases exhibiting a spectrum of $\mathrm{AD}$-type neuropathology were used to allow a correlated morphometric analysis on $\mathrm{A} \beta$ and sortilin pathologies. Brains from sortilin knockout $(-/-, n=4)$, wildtype $(+/+$, $n=4)$ and C57BL/6 mice were used for the purpose of antibody validation (Ruan et al., 2016), with hemi-brains obtained after vascular rinse with cold saline then prepared for immunohistochemistry and immunoblot, respectively. The use of postmodern human brains and laboratory animals was approved by the Ethics Committees of Central South University 
Xiangya School of Medicine and Chinese Academy of Medical Sciences, Institute of Basic Medical Sciences (human brain study), and the Animal Ethic Committee of SA Pathology at Adelaide, Australia (mouse brain study), in compliance with the Code of Ethics of the World Medical Association (Declaration of Helsinki) and the National Institutes of Health Guide for the Care and Use of Laboratory Animals.

\section{Tissue Processing}

Postmortem human brains were bisected and cut into $\sim 2 \mathrm{~cm}$-thick frontal slices. Slices from one hemisphere (opposite to handedness) were fixed in formalin for at least 1 week, with the slices from the other hemi-brain stored at $-70^{\circ} \mathrm{C}$. Temporal, frontal, parietal and occipital lobe blocks were then sampled from the fixed hemisphere, and either embedded with wax or placed in $30 \%$ sucrose in $0.01 \mathrm{M}$ phosphate buffer, which were further prepared into paraffin ( $5 \mu \mathrm{m}$ thick) or cryostat ( $40 \mu \mathrm{m}$ thick) sections. The cryostat sections were collected in phosphatebuffered saline (PBS, $0.01 \mathrm{M}, \mathrm{pH}$ 7.2), with consecutive sections placed orderly into 24 wells each containing $4-6$ sections/block with equal distance $(24 \times 40 \approx 1000 \mu \mathrm{m})$. The sections were rinsed with $\mathrm{PBS}$ twice to remove the embedding medium, and stored in a cryoprotectant at $-20^{\circ} \mathrm{C}$ before use. Mouse brains were dissected out after vascular perfusion with cold saline, with hemi-brains either snap-frozen for biochemical study or fixed by immersion in $4 \%$ paraformaldehyde for anatomical study.

\section{Immunohistochemistry and Immunofluorescence}

Four sets of consecutive mid-hippocampal temporal lobe sections from each brain, 4-6 cases together in each experiment, were stained immunohistochemically with four antibodies: goat anti-sortilin extracellular domain (diluted at 1:2000, AF3154, immunogenic peptide corresponding to amino acid (a.a.) 76-753 of human sortilin, AF3154-SP, R\&D Systems China Co. Ltd., Shanghai, China), rabbit anti-sortilin intracellular C-terminal domain (1:2000, ab16640, immunogenic peptide corresponding to a.a. 800-831 of human sortilin, ab16686, Abcam Trading Shanghai Company Ltd., Shanghai, China), monoclonal mouse anti-A $\beta$ 6E10 (1:5000, \#39320, Signet Laboratories Inc., Dedham, MA, USA) and rabbit anti-phosphorylated tau (1:5000, T6819, Sigma-Aldrich, St. Louis, MO, USA). In this set of experiments, the sections subjected to $6 \mathrm{E} 10$ labeling were invariably pretreated with formic acid for $1 \mathrm{~h}$ at room temperature. To determine the effect of several section pretreatments, including sortilin immunogenic peptide blocking (at 5 and 10 times of primary antibody concentration), heating $\left(65^{\circ} \mathrm{C}\right)$, formic acid $(100 \%)$ and guanidine hydrochloride $(\mathrm{HCl} ; 5 \mathrm{M})$ treatments, consecutive sections from four brains with extensive amyloid pathology were selected for each treatment paradigm. Also, in each experiment, several adjacent sections were processed together with other sections, excluding the primary antibody in the incubation buffer, which were used to obtain the cutoff level of nonspecific labeling in densitometry.

Other than the above specifications, all sections were treated free-floating first with $5 \% \mathrm{H}_{2} \mathrm{O}_{2}$ in $\mathrm{PBS}$ for $30 \mathrm{~min}$ and $5 \%$ normal horse serum in PBS with $0.3 \%$ Triton X-100 for $1 \mathrm{~h}$, followed by incubation with the primary antibodies at $4^{\circ} \mathrm{C}$ overnight. The sections were then reacted with biotinylated horse anti-mouse, rabbit and goat IgGs at 1:400 for $1 \mathrm{~h}$ and $\mathrm{ABC}$ reagents (1:400; Vector Laboratories, Burlingame, CA, USA) for $1 \mathrm{~h}$, with the immunoreactivity visualized in $0.003 \%$ $\mathrm{H}_{2} \mathrm{O}_{2}$ and $0.05 \%$ 3,3'-diaminobenzidine. Immunolabeling on paraffin sections was processed on-slide following dewaxing, rehydration and the antibody incubation steps described above, which involved $A \beta$, p-Tau, and sortilin immunolabeling with the rabbit antibody. The immunolabeled sections were used for the scoring of the $\mathrm{AD}$ type-pathology (i.e., $\mathrm{ABC}$ staging for amyloid pathology and Braak's I-VI staging for tau pathology (Braak et al., 2006; Montine et al., 2012), and for an overall assessment for the presence of extracellular sortilin labeling relative to the occurrence of $A \beta$ deposition.

Double immunofluorescence was initiated with section pretreatment in PBS containing 5\% donkey serum for $30 \mathrm{~min}$. The sections were then incubated overnight at $4^{\circ} \mathrm{C}$ with the rabbit sortilin antibody (ab16640, 1:1000), together with one of the following: (1) goat anti-sortilin (AF3154, 1:1000); (2) 6E10 (1:4000); (3) mouse anti-SYN (1:1000; MAB329, Millipore, MA, USA); (4) mouse anti-phosphorylated tau (PHF1, courtesy of Dr. P Davis, 1:4000); (5) mouse anti-glial fibrillary acidic protein (GFAP, 1:2000; MAB360, Millipore); and (6) mouse anti-ApoE (1:1000, NE1004, Millipore). Immunofluorescence was visualized with Alexa Fluor ${ }^{\circledR} 488$ and Alexa Fluor ${ }^{\circledR}$ 594 conjugated donkey anti-mouse, anti-rabbit or anti-goat IgGs (1:200, Invitrogen, Carlsbad, CA, USA). The sections were treated with $0.1 \%$ Sudan black to block autofluorescence before microscopic examination.

\section{Western Blot}

Preparation of tissue lysates and electrophoresis were carried out with caution to minimize the effect of temperature on protein degradation. Neocortex from the middle temporal gyrus of the frozen human cerebral hemispheres and frontal cortex from frozen mouse brains were sampled in the chamber of cryostat, then homogenized on ice by sonication in T-PER extraction buffer (Pierce, Rockford, IL, USA) containing protease inhibitors (Roche, Indianapolis, IN, USA). Resulting lysates were centrifuged at $15,000 \mathrm{~g}$ at $4^{\circ} \mathrm{C}$, with supernatants collected and protein concentrations measured by DC protein assay (Bio-Rad Laboratories, Hercules, CA, USA). Extracts containing equal amount of total protein were run in SDS-polyacrylamide gel electrophoresis (PAGE) gels (protein loading and SDS concentration to be detailed along with result description). Separated protein products were electrotransferred to Trans-Blot pure nitrocellulose membranes, which were immunoblotted with the aforementioned antibodies (rabbit and goat anti-sortilin diluted at 1:2000, 6E10 at 1:4000, BACE1 at 1:2000 and phosphorylated tau at 1:2000; Cai et al., 2010), and that for loading controls including $\beta$-tubulin-III (1:5000, Millipore), $\beta$-actin (1:5000, Millipore) or glyceraldehyde-3-phosphate dehydrogenase (GAPDH; 1:5000, Millipore). The membranes were further reacted with HRP-conjugated secondary antibodies (1:20,000; Bio-Rad Laboratories). Immunoblotting signal was 
visualized with the ECL-Plus detection kit, followed by X-ray film exposure and image capture in a laser scanner.

\section{Imaging, Data Analysis and Figure Preparation}

Immunolabeled sections were examined on an Olympus BX51 microscope and a Nikon confocal microscope equipped with digital imaging systems (CellSens Standard and Nikon-EZC1, respectively). Light microscopic images were taken using $2 \times$ to $40 \times$ objectives, with low magnification images montaged to re-construct the labeling over the temporal lobe regions. Confocal images were taken at $20 \times$ and $40 \times$ through three scans covering $\sim 6 \mu \mathrm{m}$ tissue depth. Images prepared for densitometry were obtained from comparable locations in adjacent sections using the same light intensity and digital exposure pre-settings, for all cases and all types of immunolabeling. Quantification of immunolabeled profiles was carried out following incorporation of region-matched images into a single file, which was converted into gray-scale TIFF format. Subsequently, either the areas or optic densities of the profiles/anatomic regions of interest were measured with the OptiQuant software (Packard Instruments, Meriden, CT, USA). Optic densities over immunoblotted protein bands were obtained with the same software.

All image data were exported into Excel spreadsheets and re-arranged according to groups, with specific densities calculated by background subtraction, and areal fractions or relative density levels calculated against internal references, whenever applicable. Finally, all data were entered into Prism spreadsheets, analyzed statistically, and graphed if applicable (GraphPad Prism 4.1, San Diego, CA, USA). Statistical analyses were conducted using either paired two-tailed $t$-test or a nonparametric test (Kruskal-Wallis with Dunn's multiple comparison) with the GraphPad Prism software. Details regarding group pairing, sample size and statistics for individual tests will be incorporated in the following sections along with result description as well as in figure legend. The minimal significant level of difference was set at $p<0.05$. Figures were assembled with Photoshop 7.1.

\section{RESULTS}

\section{Antibodies Against Sortilin Extracellular and Intracellular Domains Displayed Identical Neuronal Labeling in Adult Rodent and Human Cerebrum}

We validated the specificity of the sortilin antibodies to the extracellular (goat) and intracellular C-terminal (rabbit) parts using sortilin knockout $(-/-)$ and wildtype $(+/+)$ mouse brains (Figures 1A-E; Ruan et al., 2016). Both antibodies marked a $\sim 100 \mathrm{kDa}$ band in immunoblot of sortilin $+/+$, but not $-/-$, lysates, representing a presence of the full-length protein in the wild-type but not knockout mouse tissues (Figures 1B,D). By extending the film exposure time, two bands at $\sim 15 \mathrm{kDa}$ and $\sim 40 \mathrm{kDa}$ became visible in the immunoblot with the C-terminal antibody of the sortilin+/+, but not sortilin-/-, lysates
(Figure 1D), indicating the existence of specific sortilin-derived peptides in the former preparation at very low concentration. In immunohistochemistry, the two antibodies exhibited identical, essentially neuronal, labeling in the sortilin $+/+$ mouse forebrain (Figures 1C,E), whereas the labeling appeared background like in sortilin-/- counterpart (Figures 1B,D). We also confirmed a fully co-localized immunofluorescent labeling by the two antibodies, occurring primarily in the somata and dendrites of cortical and hippocampal pyramidal neurons as well as the granule cells of dentate gyrus (DG) in wild-type rodents, including at granular profiles inside the somata and large dendrites (Figures 1F-M).

The two sortilin antibodies also exhibited identical labeling pattern in mid-age human cerebrum (Figure 2). Thus, immunolabeling in the neocortex occurred in the gray matter, mainly over layers II-VI and heaviest in layers VI, with little reactivity in the WM (Figures 2A,H). At higher magnifications, pyramidal and polymorphic neurons in layers $\mathrm{V} / \mathrm{VI}$ and II/III were among the most distinctly labeled (Figures $\mathbf{2 A}, \mathbf{B}, \mathbf{D}$ ). The somata and apical dendrites of the subicular and hippocampal pyramidal neurons were clearly visualized, with granular elements visible in the cytoplasm (Figures 2C,E). In the DG, both the granule cell layer (GCL) and molecular layer (ML) showed heavy reactivity (Figures 2A,F). Hilar mossy cells and CA3 pyramidal neurons, including their dendritic trees, were well-labeled (Figures 2A,F,G,H,J), with the thorny excrescences (TE) on the dendrites clearly identifiable at high magnifications (Figures 2F,G,I,J). Overall, axonal profiles were not identifiable around the somata of cortical and hippocampal neurons (Figures 2D,E), nor in the cerebral WM (Figures 2A,H) and in the mossy fiber terminal field (Figures 2A,F,H).

\section{Sortilin C-Terminal Antibody Labeled Plaque-Like Lesions in Aged and AD Human Cerebrum}

The sortilin antibodies against the extracellular and intracellular domains exhibited sharply different labeling patterns in the brains with $\mathrm{AD}$-type pathology. Thus, the C-terminal antibody visualized plaque-like lesions, whereas the extracellular domain antibody did not (Figures 3A-H; Supplementary Figures S1, S2). Notably, in the samples with extensive plaque lesions, the neuronal labeling by the C-terminal antibody became less impressive, compared to that by the extracellular domain antibody (Figures 3A-D; Supplementary Figures S1, S2). This phenomenon appeared to likely reflect a competitive situation among the antigen epitopes in the section to bind the available antibody, because we found that the neuronal labeling could be enhanced by raising the concentration (from 1:2000 to 1:1000 or 1:500) of the rabbit antibody in the incubation buffer (data not shown).

We carried out an overall assessment of the C-terminal antibody labeling in sections from more than 70 brains with and without amyloid and tau pathology (including 10 Caucasian AD cases; Supplementary Table S1). Overall, the plaque-like sortilin labeling was found consistently among the cases that also had cerebral amyloid pathology. Thus, as assessed 

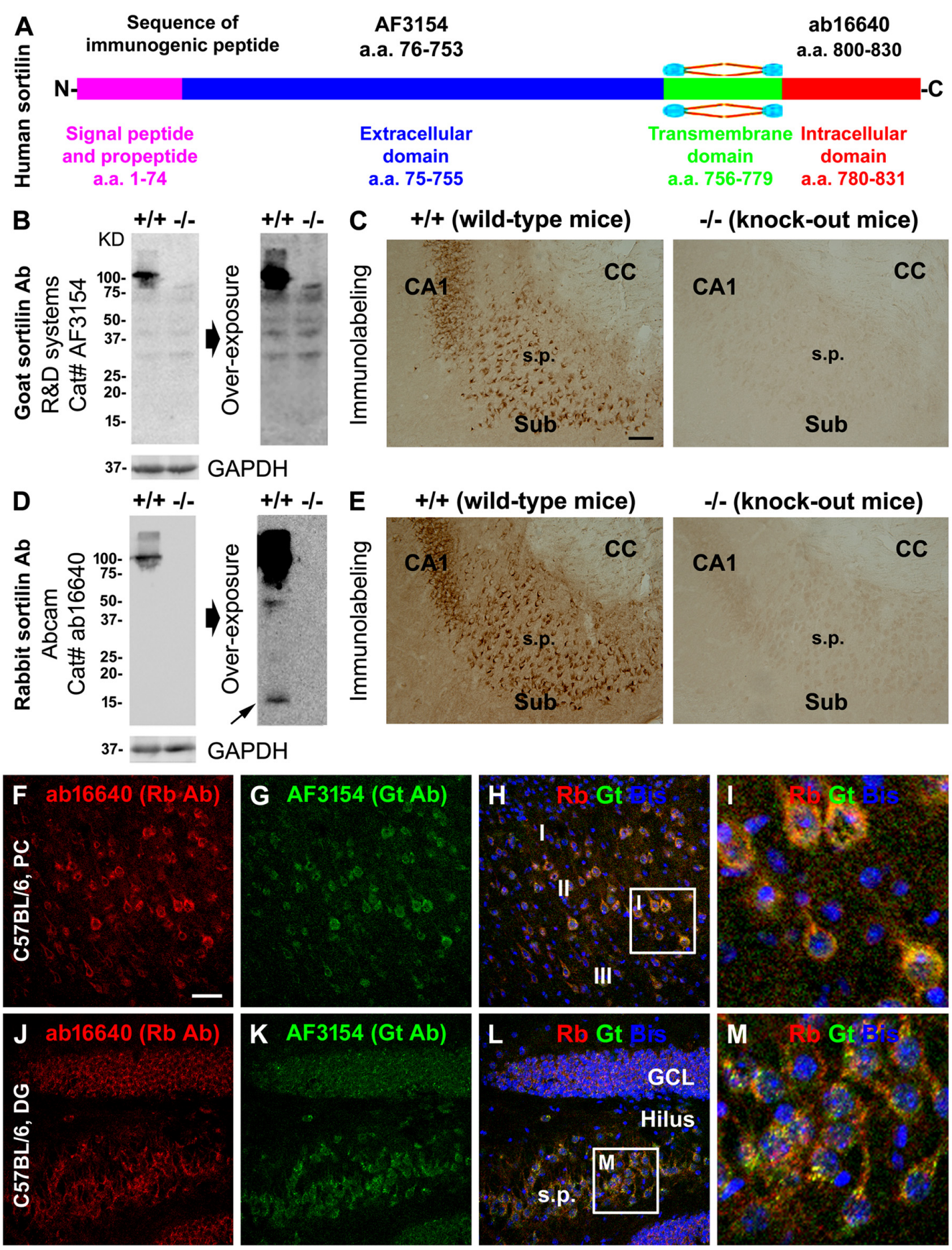

FIGURE 1 | Validation of sortilin antibodies using sortilin knockout (-/-) and wildtype (+/+) mouse brains, with a characterization of the normal expression pattern of sortilin in rodent cerebrum. Panel (A) is a schematic drawing of the human sortilin protein, with the extracellular, transmembrane and intracellular domains of varying lengths of amino acid (a.a.) residues as marked. The immunogenic synthetic peptide sequences for the extracellular and intracellular C-terminal domain antibodies are also provided. Panels $\mathbf{( B , C )}$ show representative immunoblot and immunolabeling results obtained with the goat antibody (Gt Ab) against the extracellular domain in sortilin $+/+$ and $-/-$ brains. Panels (D,E) show the results obtained with the rabbit antibody (Rt Ab) against the intracellular C-terminal. Both antibodies label a $\sim 100 \mathrm{kDa}$ band in sortilin $+/+$, but not in $-/-$, lysates (B,D). Several non-specific bands are visible in the immunoblot with the goat antibody, equally present in the sortilin $+/+$ and $-/$ - lysates, by extending the time of film exposure (B). With overexposure, two light bands at $\sim 40$ and $\sim 15 \mathrm{kDa}$ (pointed by arrow) are also noticeable in the immunoblot of sortilin $+/+$ lysates with the rabbit antibody (D). Light microscopic images (C,E) show neuronal profiles in the subiculum (Sub) to CA1 transitional region labeled by both antibodies in sortilin $+/+$, but not in $-/-$, brain sections. Confocal immunofluorescent images show completely colocalized labeling by the two antibodies in cortical pyramidal-like neurons (F-I), CA3 pyramidal neurons and granule cells of the dentate gyrus (DG) (J-M) in C57BL mouse brain, with granular elements seen intracellularly $(\mathbf{I}, \mathbf{M})$. Western blot applications: $12 \%$ SDS-PAGE gel and $13 \mu \mathrm{g}$ equal amount protein loading. Additional abbreviations: CC, corpus callosum; s.p., stratum pyramidale; GCL, granule cell layer; PC, Parietal cortex; I-III, cortical layers; GAPHD, glyceraldehyde-3-phosphate dehydrogenase. Scale bar = $200 \mu \mathrm{m}$ in (C) applying to (E); $100 \mu \mathrm{m}$ in (F) applying to (G,H,J-L), equivalent to $25 \mu \mathrm{m}$ for (I,M). 

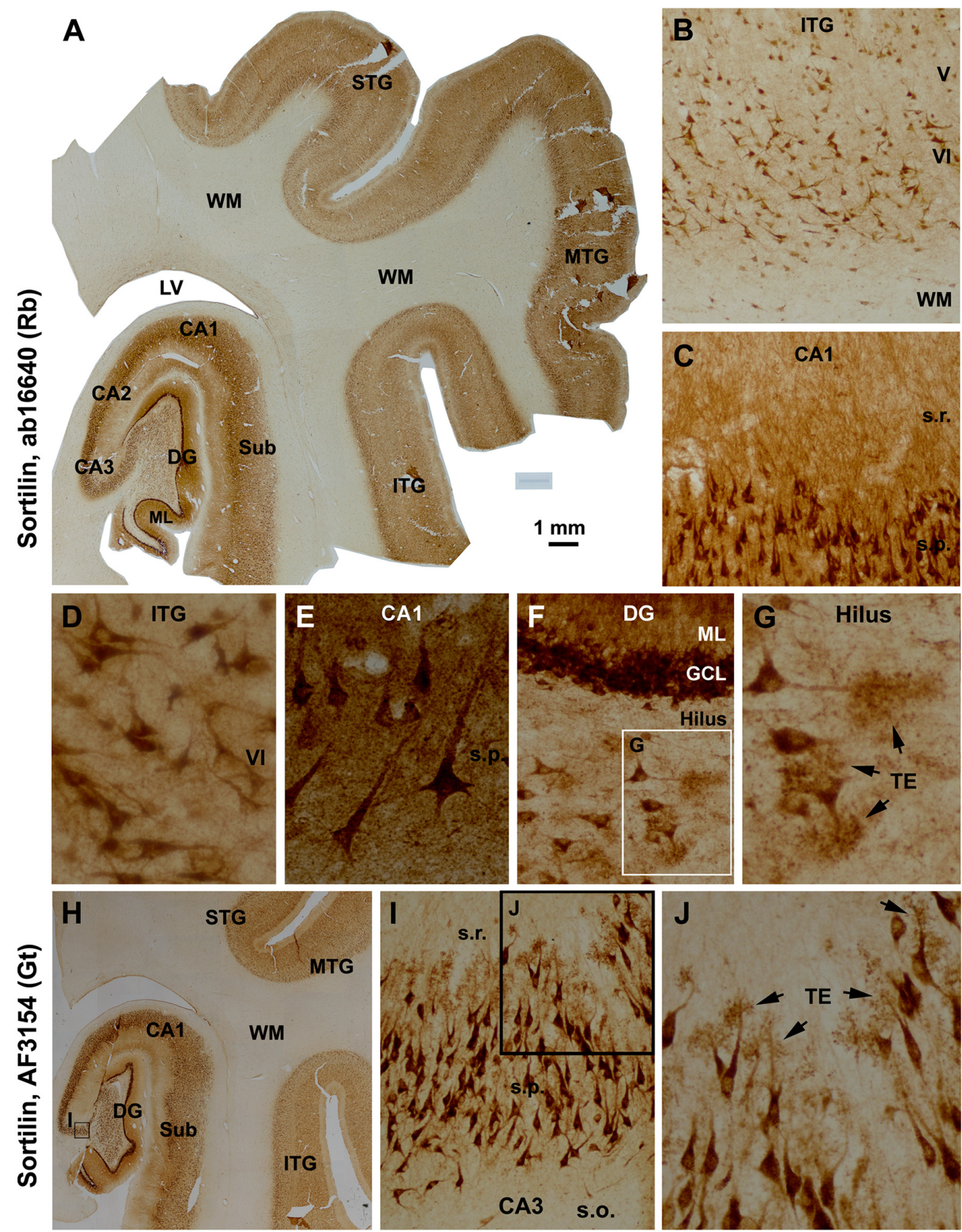

FIGURE 2 | Characterization of the normal sortilin expression pattern in the human cerebral neocortex and hippocampal formation with tissue from a mid-age case. Panel (A) is a montaged low magnification image illustrating the expression of sortilin labeled with the rabbit antibody across the superior, middle and inferior temporal gyri (STG, MTG, ITG), subiculum (Sub), hippocampal Ammon's horn (CA1-3) and DG. The labeling occurs in the gray matter but barely in the white matter (WM). The molecular layer $(\mathrm{ML})$ of the DG shows strong neuropil reactivity. Panels $(\mathbf{B}, \mathbf{D})$ show higher power views of the labeling in the neocortex, with large and middle-sized pyramidal and polymorphic neurons exhibiting strong immunoreactivity. Panels $\mathbf{( C , E ) ~ s h o w ~ h e a v y ~ l a b e l i n g ~ i n ~ C A 1 ~ p y r a m i d a l ~ n e u r o n s ~ s p e c i f i c a l l y ~ i n ~ t h e ~ s o m a t a ~ a n d ~}$ dendrites, with the stratum radiatum (s.r.) also exhibiting strong reactivity in fine dendritic processes and neuropil. Panels (F,G) are higher magnification images of the DG; the somata and proximal dendrites of the granule cells are intensively stained. The somata and dendrites of hilar mossy cells are distinctly labeled, with the thorny excrescences (TE, arrows) well-displayed. Axons of the cortical and CA1 pyramidal neurons are not visible (D,E), with no labeling seen in the mossy fiber terminal field (A,F). Panel $\mathbf{( H )}$ shows that the goat antibody exhibits essentially the same neuronal labeling pattern in the hippocampal formation and temporal neocortex as with the rabbit antibody (A), with enlarged views specifically illustrating the visualization of the TE on the dendrites of the CA3 pyramidal neurons (I,J). Additional abbreviations: IV-VI, cortical layers. Scale bar $=1 \mathrm{~mm}$ in (A), equivalent to $2 \mathrm{~mm}$ for (H), $200 \mu \mathrm{m}$ for (B), $100 \mu \mathrm{m}$ for (C,I), $50 \mu \mathrm{m}$ for (D,F,J) and $25 \mu \mathrm{m}$ for (E,G). 

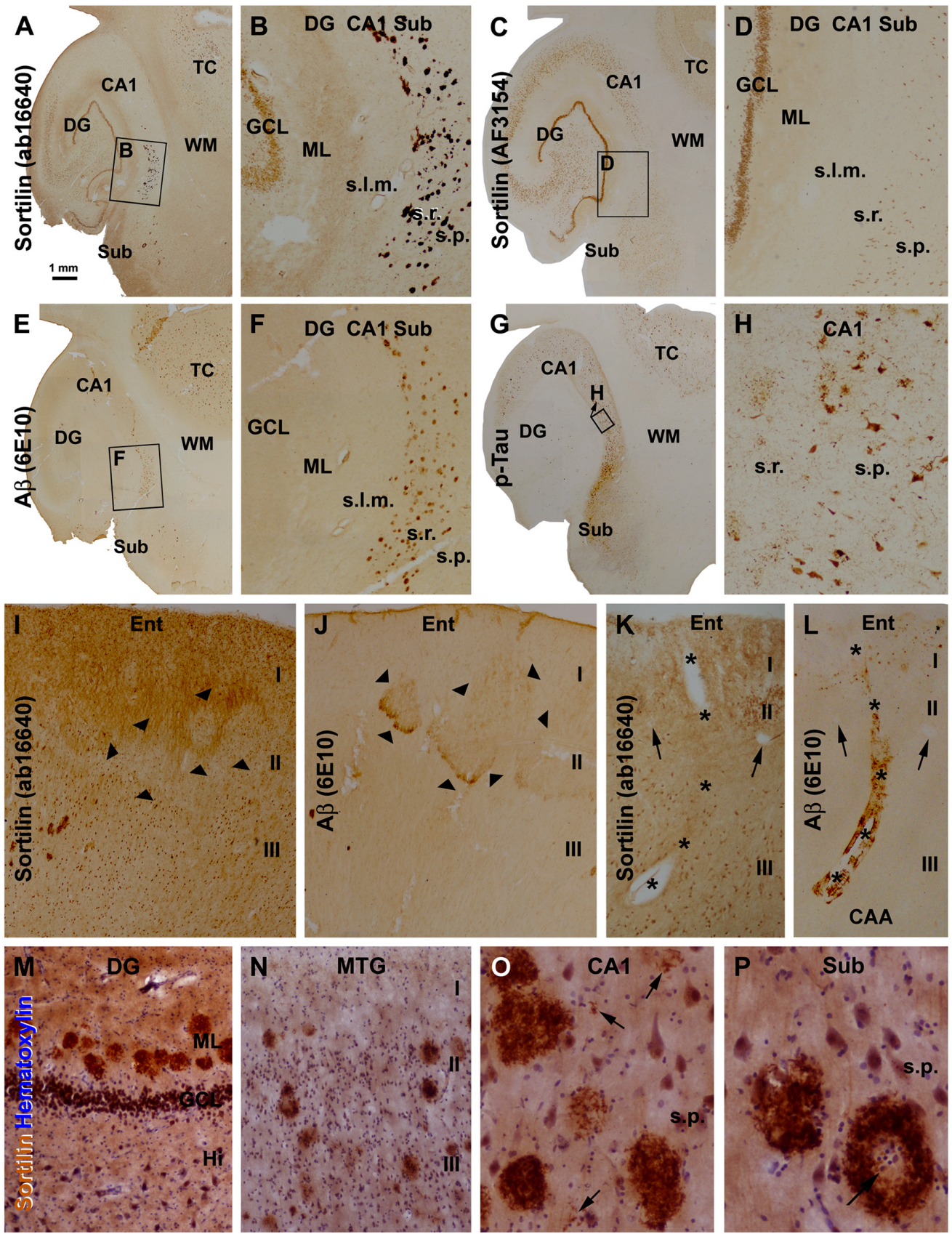

FIGURE 3 | Morphological characterization of sortilin labeled plaque lesions relative to amyloid and tau pathology using sections from cases with Alzheimer's disease (AD). Panels (A-G) show low and high power (framed areas) views over the hippocampal formation and part of temporal cortex (TC) from adjacent sections, with the 4 markers as indicated. The rabbit sortilin antibody labels plaque lesions that are regionally matched to that labeled by the $6 \mathrm{E} 10 \beta$-amyloid $(\mathrm{A} \beta)$ antibody $(\mathbf{A}, \mathbf{B}, \mathbf{E}, \mathbf{F})$. Both the rabbit and goat sortilin antibodies label neuronal profiles seen clearly in the GCL at low magnification (a-d). Immunolabeling of phosphorylated Tau ( $p$-Tau) is seen in tangled neurons and neuritic plaques $\mathbf{( G , H ) . ~ P a n e l s ~}(\mathbf{I}, \mathbf{J})$ are images taken from adjacent sections of the entorhinal cortex (Ent) from another AD case, showing a lack of extracellular sortilin labeling at the layer II cell islands (pointed by arrowheads) that exhibited diffuse A $\beta$ deposition. Panels (K,L) shows a lack of sortilin deposition in association with cerebral amyloid angiopathy (CAA), also as assessed between consecutive sections. Asterisks denote the trajectory of a longitudinally cut amyloid arteriole entering the cortex from pia surface, with the two arrowheads pointing to two cross-sectioned normal vessels, for additional spatia reference. Panels (M-P) show high magnification views of extracellular sortilin deposits in sections counterstained with hematoxylin. Plaques occur in the ML of the DG in a row (M), and in the cortex of the middle temporal gyrus (MTG) with different sizes and labeling intensity (N). Panel (O) shows the occurrence of small amount of deposits (pointed by arrows) in the neuropil in areas about the sizes of the cell nuclei and neuronal somata, along with densely packed mature-looking plaques. Panel (P) shows circular plaques with cell nuclei (arrows) in the center. Additional abbreviations: I-III, cortical layers; s.I.m., stratum lacunosum-moleculare; other abbreviations are as defined in Figure 1. Scale bar = $1 \mathrm{~mm}$ in (A) applying to (C,E,G); equivalent to $200 \mu \mathrm{m}$ for (B,D,F,I,J), $50 \mu \mathrm{m}$ for (K-N) and $25 \mu \mathrm{m}$ for (O,P). 
between consecutive temporal lobe sections, sortilin and $\mathrm{A} \beta$ labeled plaques both occurred over the neocortex, subiculum and hippocampal areas with their distributions matchable in reference to given subregions and lamina, noticeable at low magnification (Figures 3A,B,E,F; Supplementary Figures S1, S2). As with $A \beta$ plaques, sortilin plaques lacked a spatial parallelism with tau pathology over the temporal lobe regions (Figures 3A-H; Supplementary Figures S1, S2) (therefore we did not quantitatively analyze sortilin relative to tau pathology). While assessing the overall pattern of extracellular sortilin/A $\beta$ labeling, it should be notified that, along with the compact-like lesions diffuse $\mathrm{A} \beta$ deposition was present in local areas of the cortex, e.g., around layer I, the cell islands in layer II of the entorhinal cortex and the WM. In comparison, immunoreactivity of the C-terminal sortilin antibody in the above anatomic locations appeared to be not changed (relative to adjacent regions; Figures 3I,J; Supplementary Figures S2F,I). Also as a general note, 6E10 was found to label $\beta$-amyloidosis locally around the pia and blood vessels in the gray and WMs, whereat no increased extracellular sortilin deposition was found at these locations (Figures 3K,L).

At high magnifications more clearly in immunolabeled sections with hematoxylin counterstain, the plaque-like lesions detected with the sortilin C-terminal antibody consisted of extracellularly deposited fibrillary products (Figures $3 \mathbf{M}-\mathbf{P}$ ). The plaques were largely round, varying in size, often with the fibrillary material densely packed across the entire plaque. However, some plaques contained less densely stained fibrils (Figure 3N). Coexisting with the above, labeled extracellular fibrils were seen to deposit over small areas about the sizes of the neuronal nuclei, or as mini-plaques of the sizes of the neuronal perikarya (Figure 30). Large sortilin plaque profiles could be ring-like with the deposits packed on the periphery, leaving an empty or pale center that sometimes occupied by cell nuclei (Figure 3P).

We used immunogenic peptide absorption to verify the specific labeling of the sortilin antibodies in the human brain sections. The labeling of the rabbit antibody was diminished to the background levels (defined using sections processed in the absence of primary antibody), when the immunohistochemistry was proceeded by co-incubation with the C-terminal immunogenic peptide at either 5 (data not shown) and 10 times of the concentration of the primary antibody (Figures 4A,B,D,I). Such an effect did not occur by including the extracellular domain peptide in the incubation buffer (Figures 4A,C,I). Vice versa, the extracellular immunogenic peptide could completely block the labeling of the goat antibody, but did not affect the immunolabeling of the rabbit C-terminal antibody (Figures 4E-H,J).

\section{Sortilin and A $\beta$ Deposition at Senile Plaques were Anatomically Correlated in Human Cerebrum}

In a subset of the aged and $\mathrm{AD}$ cases we noticed sortilin/ $\mathrm{A} \beta$ plaques preferentially distributed along the ML of the DG, with distinctly labeled plaques also present in the subiculum and adjoining CA1 area (Figures 3K, 5). Therefore, we carried out correlative areal ratio analyses of sortilin and $A \beta$ plaques across the ML and in a defined area of the CA1/subiculum region. The fractional areas of the sortilin and $A \beta$ plaques, expressed as $\%$ area occupied, were plotted relative to individual cases (Figures 5A,B,E). A strong positive correlation $(P<0.0001$, $R^{2}=0.932$ ) between the fractional areas of sortilin and $\mathrm{A} \beta$ plaques in the ML was found based on the analysis of sections from 20 individuals (Figure 5F). In the CA1/subiculum transitional region selected for quantification, the fractional areas of sortilin and $\mathrm{A} \beta$ plaques were also positively correlated $\left(P<0.0001, R^{2}=0.983, n=24\right)$ in a case-dependent manner (Figures 5E,F). We further assessed if the overall amounts of sortilin and $A \beta$ deposition were correlated in the neocortex, using a densitometric approach (Figure 5G). The resulting densitometric data indicated a positive correlation $\left(P<0.0001, R^{2}=0.880, n=15\right)$ between sortilin and $\mathrm{A} \beta$ deposition in reference to individual brains (Figure $5 \mathbf{H}$ ). It should be noted that the total optic density measured in a region reflected that contributed by labeled plaques as well as neurons. In the case of $A \beta$ immunolabeling, diffuse plaques and vascular $\beta$-amyloidosis present in the measured areas would also contribute to the yielded total optic density. Nonetheless, the densitometric correlation reflected a trend of overall parallelism between sortilin and $A \beta$ extracellular deposition in the neocortex.

In double immunofluorescence, only the rabbit antibody, but not the goat antibody, labeled plaques, while there was a colocalization of the labeling by the two antibodies in neuronal somata (Figures 6A1-4). Colocalization of extracellular sortilin and $\mathrm{A} \beta$ labeling at individual compact-like plaques was clearly seen in the hippocampal and cortical regions, shown for examples in the ML of the DG (Figures 6B1-4) and the subiculum (Figures 6C1-4). Notably, while the immunofluorescence appeared to be parallel in intensity between the two markers across the entire area of a plaque (Figures 6B1-4); it could be stronger for one marker than the other over subareas of the same plaque (Figures 6C1-4). Sortilin and SYN immunofluorescence co-existed at apparently neuritic plaques, with SYN-labeled DNs surrounded by or intermingled with extracellular sortilin labeling (Figures 6D1-4). Extracellular sortilin labeling was also found to coexist with neuritic elements with weak p-Tau immunofluorescence in plaques (Figures 6E1-4). Hypertrophic astrocytes with bright GFAP immunofluorescence were present in the vicinity of some sortilin labeled plaques (Figures 6F1-4). Moreover, sortilinlabeled plaques colocalized with extracellular ApoE labeling (Figures 6G1-4).

\section{A $\beta$-Enhancing Antigen Retrieval did not Affect Extracellular Sortilin Labeling}

Tissue processing and antigen retrieval, e.g., temperature, formic acid and guanidine $\mathrm{HCl}$, could affect $\mathrm{A} \beta$ aggregation or $\mathrm{A} \beta$ immunodetection. We therefore explored if sortilin labeling could be affected by some $A \beta$-enhancing antigen retrieval methods. These experiments were carried out 


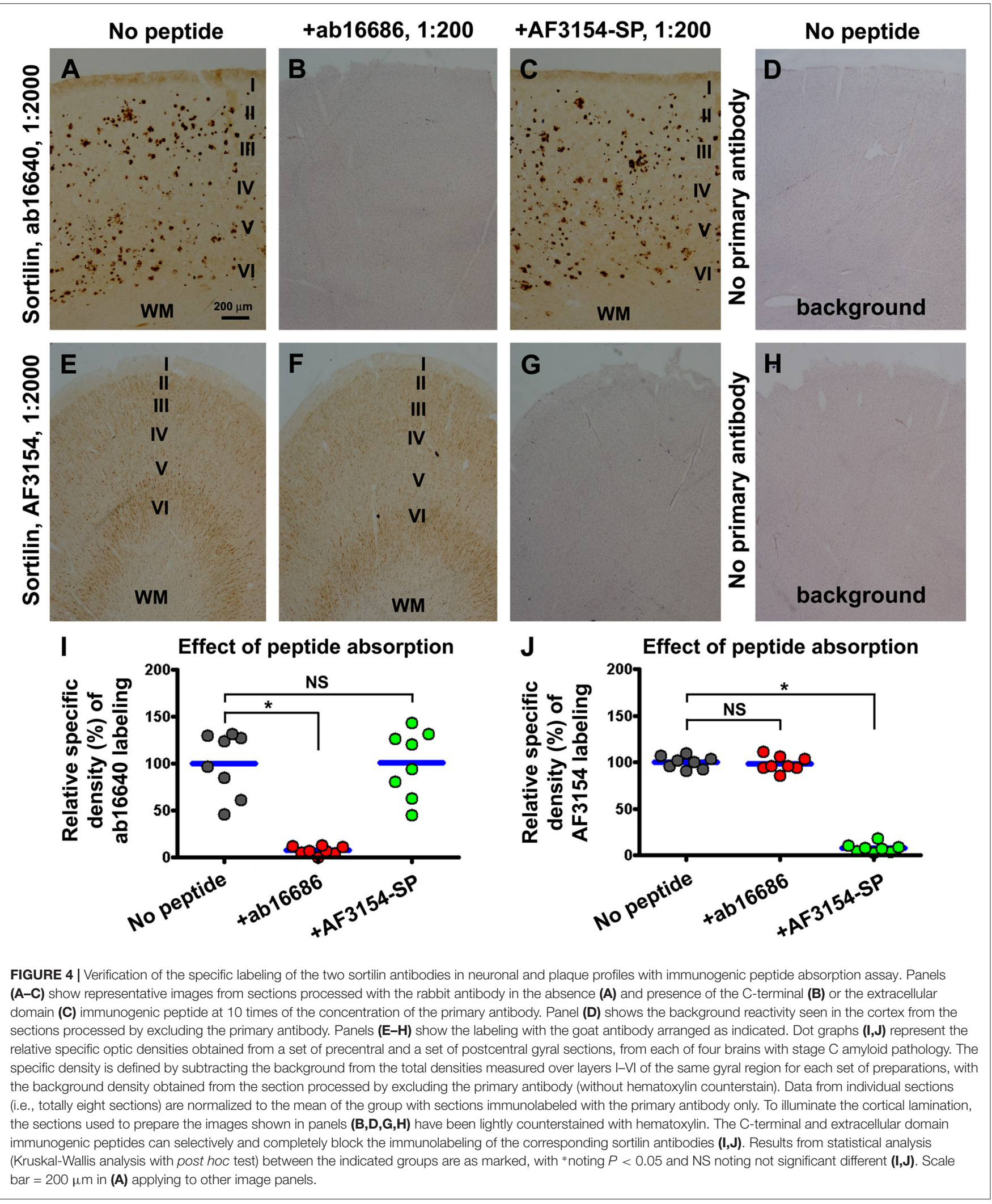

by batch-processing consecutive frontal (blocked from the precentral gyrus) and parietal (postcentral gyrus) neocortical sections subjected to untreated and pretreated conditions. The sections from four brains with stage $\mathrm{C} A \beta$ pathology were 

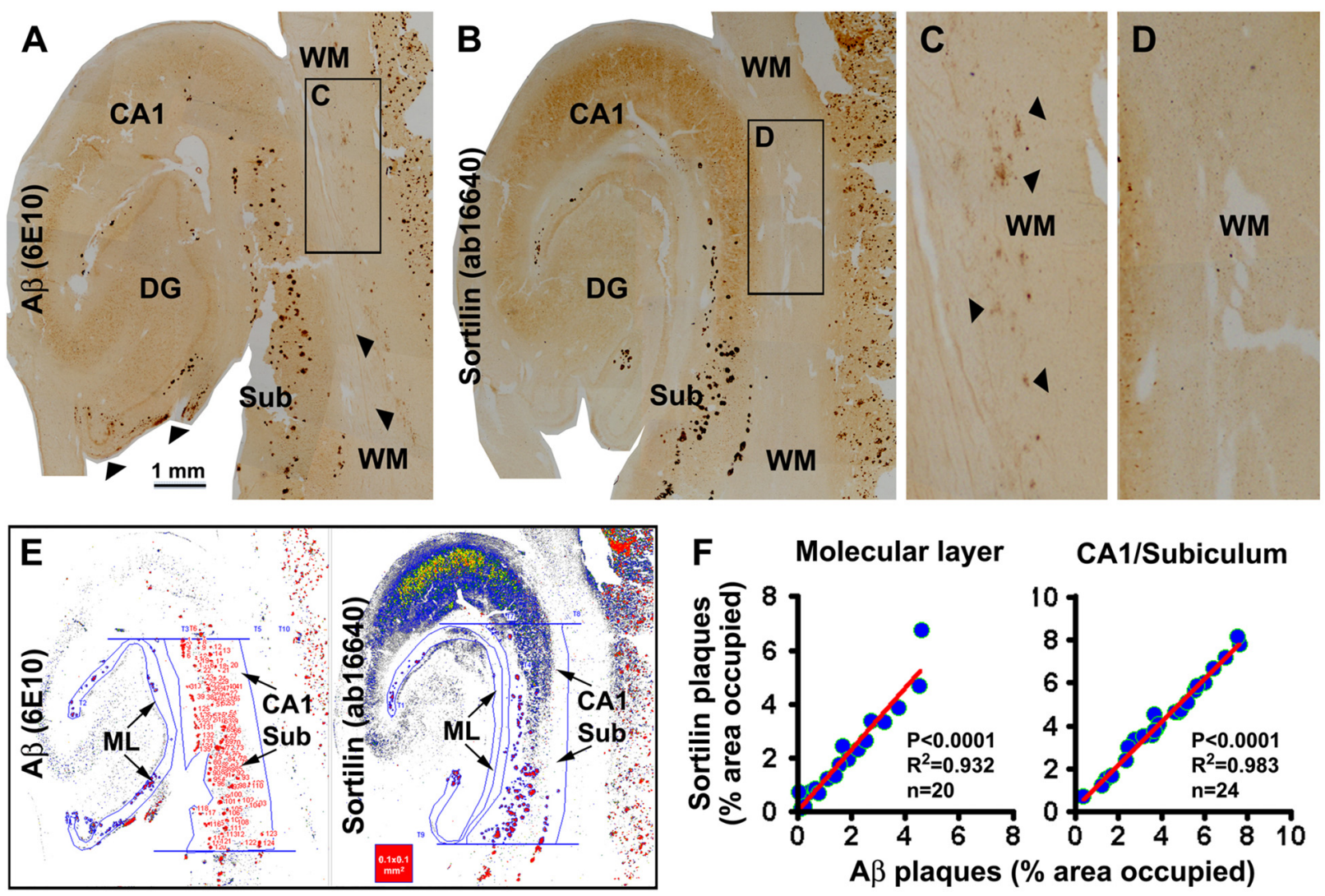

F Molecular layer

CA1/Subiculum
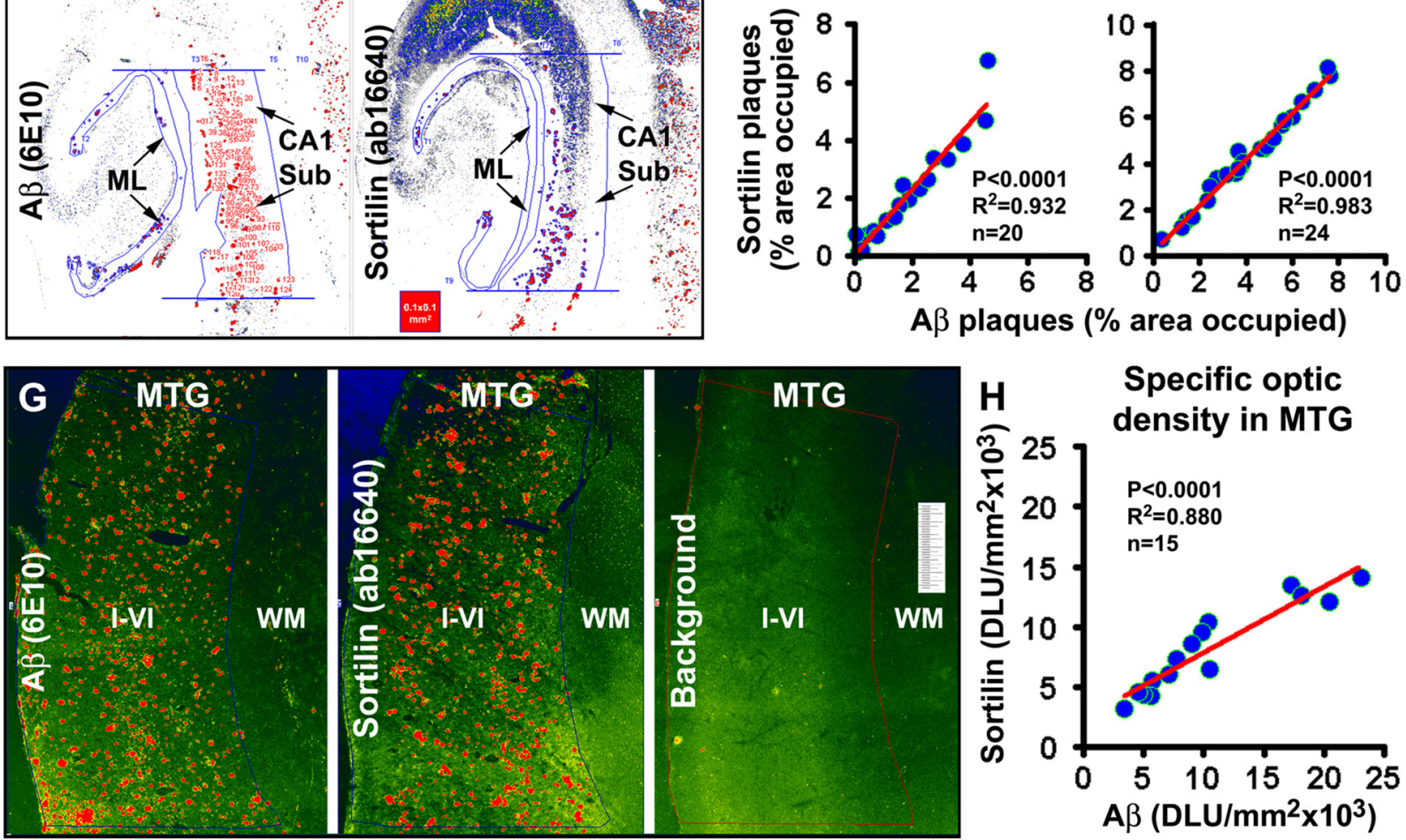

FIGURE 5 | Quantitative analysis of sortilin relative to $\beta$-amyloid deposition in hippocampal lamina and temporal neocortex. Panels $(\mathbf{A}, \mathbf{B})$ show labeling with the rabbit sortilin antibody and $6 \mathrm{E} 10$ in consecutive temporal lobe sections used for quantification. The framed WM areas are enlarged to show diffuse $A \beta$ labeling (pointed by arrows, also in panel (A) along the pia) that is not associated with extracellular sortilin deposition (C,D). The pseudocolored screen print image (E) illustrates the methodology to measure the fractional areas of sortilin and $\mathrm{A} \beta$ plaques manually over the $\mathrm{ML}$ and in the CA1/subiculum transitional region. The latter is defined by two parallel lines passing the upper and lower edges of the GCL and between the hippocampal fissure and gray/WM border. Areas of individual plaques are measured by tracing along their outer border using the irregular selecting line-drawing tool (OptiQuant software). The sum of plaque-occupied areas is divided by the total area of the reference region, yielding the fractional areal value, expressed as $\%$ of area occupied, for each case. The fractional areal values for sortilin and $A \beta$ plaques are plotted against individual aged and $A D$ cases, with sample size noted in the graphs. In both the ML and the CA1/subiculum transitional region, the fractional areas of sortilin and A $\beta$ plaques are positively correlated among the cases (F). Pseudocolored image (G) illustrates the methodology to obtain the total densities of sortilin and 6E10 labeling over the same cortical area (layers I-VI) of the middle temporal gyrus (MTG) in consecutive sections, and to obtain the background cutoff density from another adjacent section batch-processed excluding primary antibodies. Specific optic density is calculated by subtracting the background from total densities. Panel $\mathbf{( H )}$ plots specific densities of sortilin and 6E10 immunolabeling relative to individual cases analyzed, showing a positive correlation between the two measurements. Statistical results obtained via a nonparametric (Kruskal-Wallis) test are also marked in the graphs $\mathbf{( F , H )}$. Scale bar $=1 \mathrm{~mm}$ in (A) applying to (B), equal to $250 \mu \mathrm{m}$ for (C,D). 

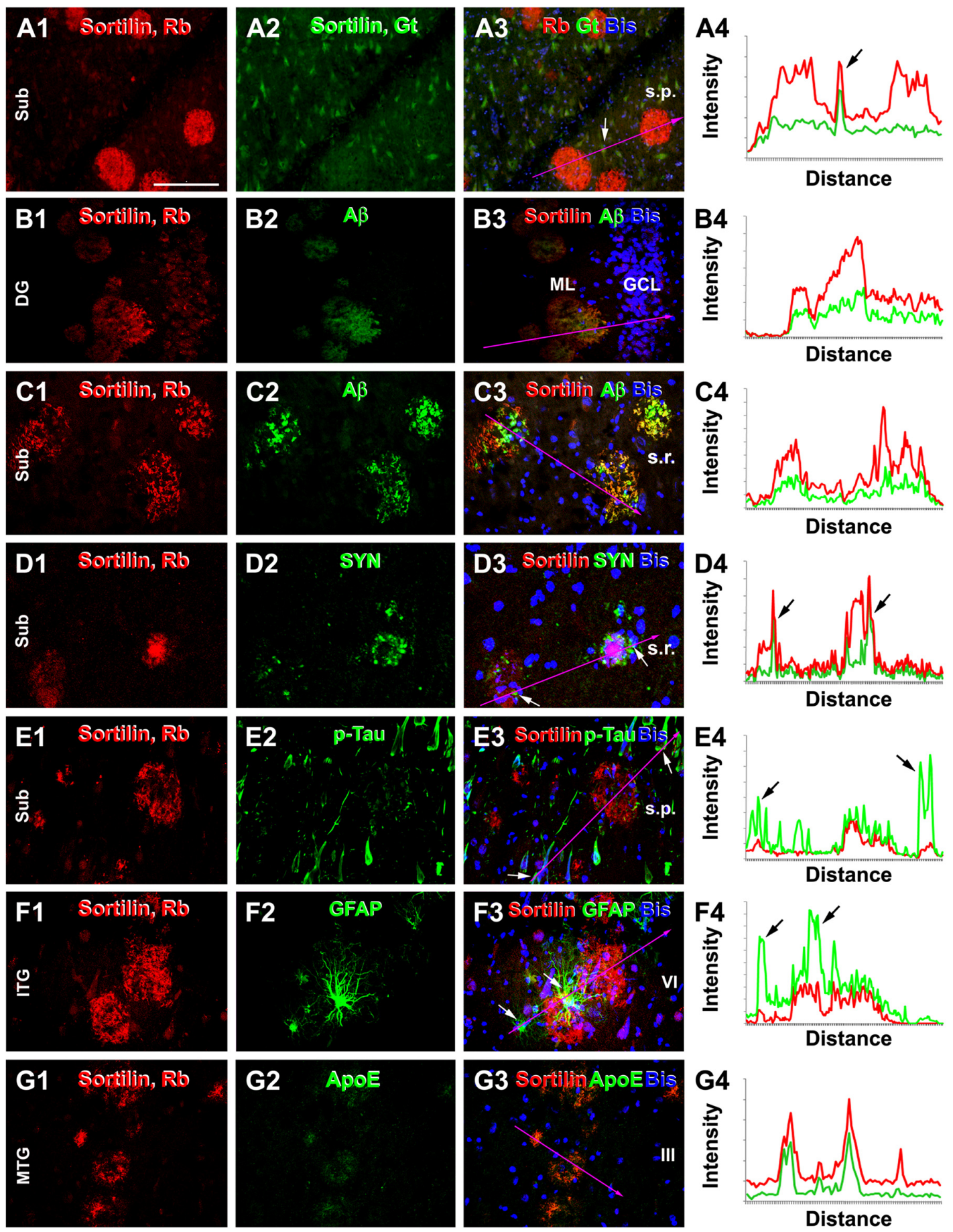

FIGURE 6 | Double immunofluorescent characterization of sortilin labeling at neuritic plaques. Each set shows the immunolabeling with the rabbit sortilin antibody relative to another marker, with bisbenzimide nuclear labeling included in the merged image. Imaged region and lamina are as indicated. Histograms illustrate the relative intensity of the red and green fluorescence measured along the line marked in the fluorescent images, with arrows pointing to the locations of labeled cellular or neuritic elements. Panels (A1-4) show a selective labeling by the rabbit sortilin antibody at plaques, while neuronal somata are labeled by both sortilin antibodies. Panels (B1-4) show sortilin and A $\beta$ labeling with parallel intensity among plaques in the ML. Panels (C1-4) show an overall colocalization of sortilin with A $\beta$ in the plaques, while the fluorescent intensity is not parallel at local areas between the two markers (merged color appears more greenish, reddish or neutrally yellow). Panels (D1-4) show sortilin labeling coexisting but not colocalizing with dystrophic neurites (DNs) with distinct synaptophysin (SYN) immunoreactivity at the same plaques. Panels (E1-4) show extracellular sortilin labeling coexisting with DNs with weak phosphorylated-Tau ( $p$-Tau) reactivity. Panels (F1-4) show sortilin plaques surrounded by hypertrophic astrocytes with bright GFAP immunofluorescence. Panels (G1-4) display colocalization of extracellular sortilin and ApoE labeling at plaques. Scale $=100 \mu \mathrm{m}$ in $\mathbf{( A )}$ applying to all image panels. 
used, with densitometric data obtained from the same gyral region.

Compared to control, sections preheated at $65^{\circ} \mathrm{C}$ for $1 \mathrm{~h}$ and $3 \mathrm{~h}$ had more intense $\mathrm{A} \beta$ immunolabeling, especially along the pia, over layer I, in the WM and at individual sites of cerebral vasculature (i.e., cerebral amyloid angiopathy, CAA; Figures 7A-C). The mean density of $\mathrm{A} \beta$ labeling measured over layers I-VI increased to $433.4 \pm 134.3 \%$ and $470.9 \pm 146.2 \%$ in the sections preheated for $1 \mathrm{~h}$ and $3 \mathrm{~h}$, respectively, relative to control (100 $\pm 37.3 \%)$, exhibiting an overall difference among the three groups $(P=0.0003$, KWI $=16.1)$, with significant difference between the control and both preheated groups, but not between the groups heated for $1 \mathrm{~h}$ and $3 \mathrm{~h}$, by post hoc test (Figure 7D). On the contrary, section preheating did not alter the pattern and intensity of sortilin labeling (Figures 7E-H).

Section pretreatment with formic acid for $1 \mathrm{~h}$ and $3 \mathrm{~h}$ also greatly enhanced $A \beta$ labeling relative to untreated controls, with the effect readily maximized by $1 \mathrm{~h}$ treatment therefore presented below. Quantitatively, the mean density of $A \beta$ labeling in formic treated sections increased significantly $(P<0.0005$, paired $t$-test), to over 4 -fold $(433.4 \pm 134.3 \%)$ relative to untreated sections (100 \pm 32.2\%; Figures 7I,J,M). Again, formic acid treatment did not affect the distribution or intensity of sortilin labeling (Figures 7K-M). Pretreatment of sections with guanidine $\mathrm{HCl}$ for either $1 \mathrm{~h}$ or $3 \mathrm{~h}$ (not shown) appeared to enhance $A \beta$ labeling slightly (however, not reached significance), relative to untreated control (Figures $\mathbf{7 N}, \mathbf{O}, \mathbf{R}$ ). The distribution or the overall amount of sortilin labeling was not different between the treated and untreated sections (Figures 7P-R).

\section{Sortilin Fragments were Elevated in AD Relative to Control Human Cortical Lysates}

Western blot was used to explore the putative sortilin products found deposited at neuritic plaques, with an attempt to identify potential dependent effect of the presence of amyloid pathology as well as brain aging. Thus, temporal neocortical samples ( $n=9$ /group, from the frozen hemisphere) were obtained from the cases in which the fixed contralateral hemispheres were pathological examined (see Supplementary Table S1). Cortical lysates were immunoblotted with the two sortilin antibodies, and antibodies to APP, BACE1 and p-Tau (as pathological controls). The rabbit sortilin antibody blotted the principal band migrated at $\sim 100 \mathrm{kDa}$, which appeared thicker in the lysates from the $\mathrm{AD}$ and Aged relative to Mid-age groups. Another prominent band blotted by this antibody migrated at $\sim 15 \mathrm{kDa}$, which was present in lysates from the $\mathrm{AD}$ and Age groups, denser in the former, while it was absent or minimal in the lysates from Mid-age subjects (Figure 8A). The medians of normalized (to $\beta$-actin signal) densities of the $100 \mathrm{kDa}$ band were significantly different in the $\mathrm{AD}$ (mean $\pm \mathrm{SD}=209.6 \pm 9.4 \%$, same format below), Aged (192.2 $\pm 29.3 \%)$ and Mid-age (147.2 $\pm 38.8 \%)$ groups $(P=0.0220$, Kruskal-Wallis statistic index, i.e., KWI, 7.6), with Dunn's Multiple Comparison Test reported a statistical difference between the $\mathrm{AD}$ and Mid-age groups (Figure 8E1). The median densities of the $15 \mathrm{kDa}$ product were different in the three groups $(P<0.0001, \mathrm{KWI}=21.3)$, with post hoc test indicating a significant increase in the AD (190.2 $\pm 34.7 \%)$ relative to Aged $(44.9 \pm 27.1 \%)$ and Mid-age (6.8 $\pm 7.7 \%)$ groups (Figure 8E2). A light $\sim 40 \mathrm{kDa}$ band was also visualized by the rabbit antibody in some $\mathrm{AD}$ and Aged cases, but not in the Mid-age group (Figure 8A) (not quantified as it was not consistently present). Different from the rabbit antibody, the goat sortilin antibody only blotted the $\sim 100 \mathrm{kDa}$ band (Figure 8B, quantitative data not shown), which showed a parallel trend in density between the cases as that blotted by the rabbit antibody. Immunoblotted $\mathrm{p}$-Tau products occurred in large amounts in the lysates from the $\mathrm{AD}$ cases, appearing as a smear of bands $\leq 70 \mathrm{kDa}$ (Figure 8C). The median density of the $\mathrm{p}$-Tau products was increased in the $\mathrm{AD}$ group (1455.1 $\pm 745.0 \%)$ relative to Aged group (109.4 $\pm 205.5 \%)$ and Mid-age group (2.5 $\pm 3.8 \%)$ $(P<0.0001$, KWI = 20.2) (Figure 8E3). The bands of full-length APP protein $(\sim 100 \mathrm{kDa})$ blotted with the $6 \mathrm{E} 10$ antibody appeared to be denser among the $\mathrm{AD}$ cases (Figure 7E), with an overall difference of its levels between AD (183.9 $\pm 13.2 \%)$, Aged $(151.6 \pm 27.4 \%)$ and Mid-age $(88.1 \pm 43.9 \%)$ groups $(P=0.0003$, $\mathrm{KWI}=16.0)$, elevated in the $\mathrm{AD}$ relative to the Mid-age groups by post hoc test (Figure 8E4). The amount of BACE1 protein $(\sim 70 \mathrm{kDa})$ appeared to be increased among the $\mathrm{AD}$ cases relative the other two groups (Figure 8D), with densitometry indicating an overall difference $(P=0.0003, \mathrm{KWI}=16.0)$ between the $\mathrm{AD}(138.7 \pm 15.1 \%)$, and Aged $(118.2 \pm 22.1 \%)$ and Mid-age $(105.9 \pm 16.2 \%)$ groups, and significant difference between the $\mathrm{AD}$ and Mid-age groups by post hoc test (Figure 8E5). Amounts of $\beta$-actin (used as a total protein loading control) and $\beta$-tubulin (used as a neuronal protein control) appeared to be comparable between the samples (Figure 8D).

\section{DISCUSSION}

\section{Full-Length Sortilin Is Enriched in the Somatodendritic Compartment of Cerebral Neurons}

Sortilin is a type I transmembrane protein consisted of 831 a.a. residues in Homo sapiens, with a long extracellular $\mathrm{N}$-terminal sequence (755 a.a. in length), a single-pass transmembrane domain (a.a. 756-779) and a short intracellular C-terminal tail (a.a. 780-831) (Westergaard et al., 2004; Hermey, 2009). First cloned from human brain (Petersen et al., 1997), sortilin is also called as neurotensin receptor-3 for an initially identified role in neurotensin signaling (Mazella et al., 1998; Sarret et al., 2003). Follow-up cell biology studies indicate that it plays a general role in sorting/trafficking of membrane proteins between intracellular organelles (Strong et al., 2012; Mortensen et al., 2014; Kjolby et al., 2015). Sortilin is also known as an ApoE receptor, and may play a key role in lipoprotein metabolism and involve in some cardiovascular and metabolic disorders (Carlo, 2013; Coutinho et al., 2013; Kjolby et al., 2015). In the nervous system, sortilin is shown to regulate the signaling of nerve growth factors and progranulin, which may relate to disease mechanisms underlying some neurological and mental disorders (Nykjaer et al., 2004; Chen et al., 2005; Hu et al., 2010; Yang et al., 2011, 2013). 


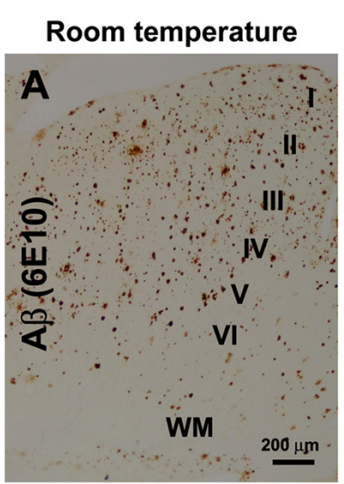

$65^{\circ} \mathrm{C}$ Heating, $1 \mathrm{hr}$
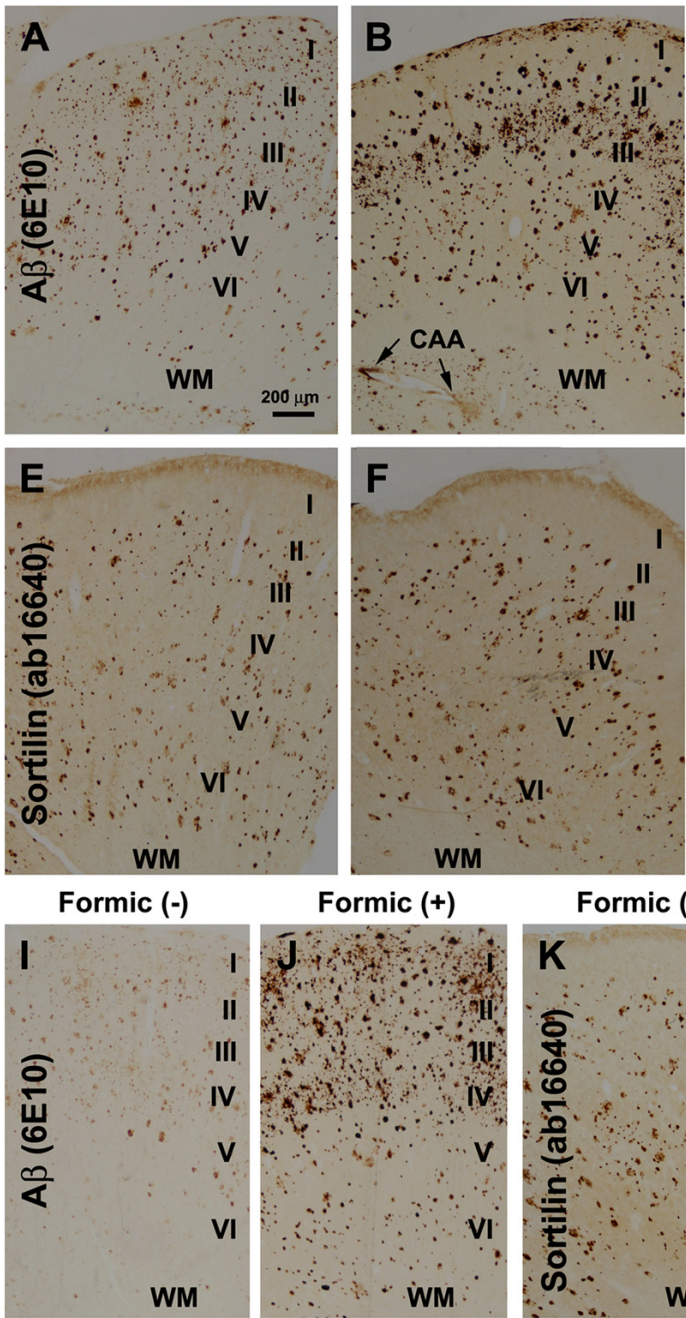

Guanidine $\mathrm{HCl}(-)$ Guanidine $\mathrm{HCl}(+)$
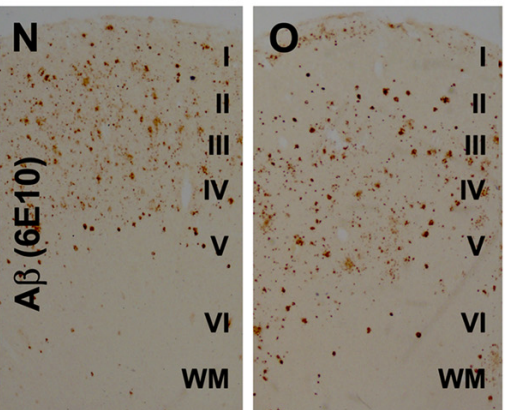

$65^{\circ} \mathrm{C}$ Heating, 3 hrs
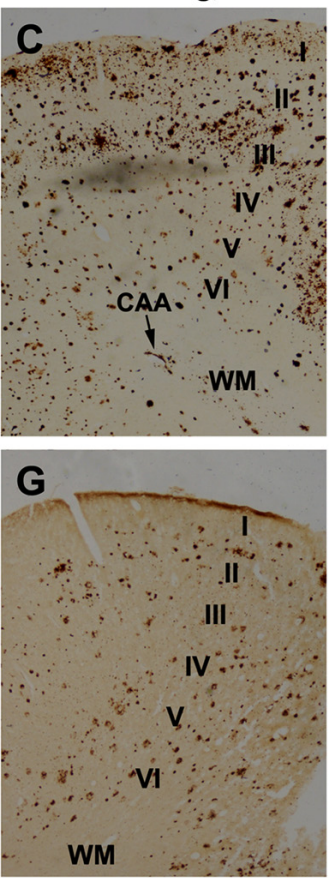

\begin{abstract}
Formic (-)
\end{abstract}

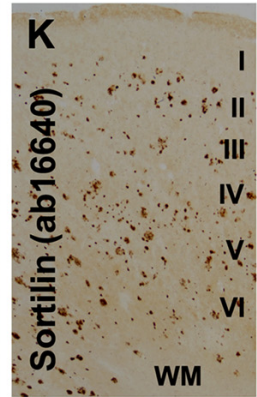

Guanidine $\mathrm{HCl}(-)$

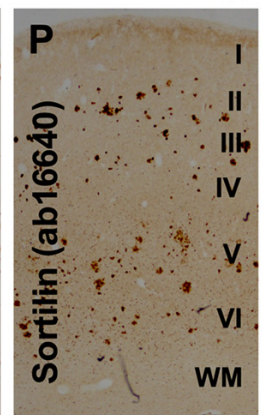

Formic (+)

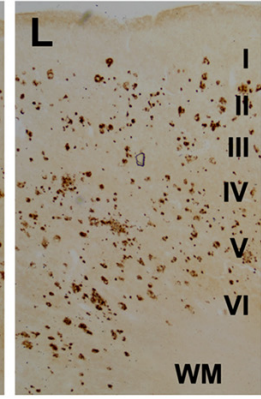

Guanidine $\mathrm{HCl}(+)$

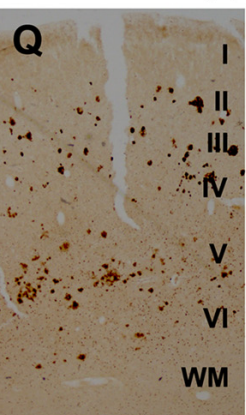

Effect of heating

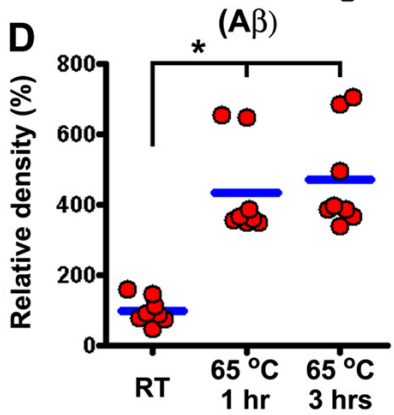

Effect of heating

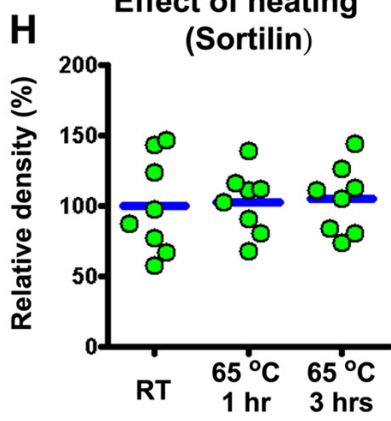

Effect of formic treatment

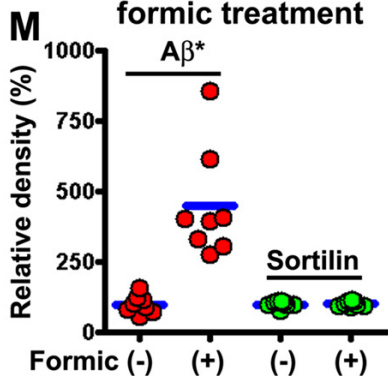

Formic (-) (+) (-) (+)

Effect of guanidine $\mathrm{HCl}$ treatment

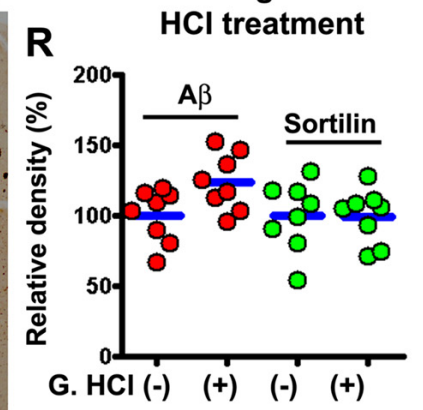

FIGURE 7 | Comparative characterization of the effect of antigen retrieval pretreatments on sortilin and A $\beta$ labeling using neocortical sections from cases with severe cerebral amyloid pathology. Adjacent sections of the precentral and postcentral gyri from four cases are first subjected to an antigen retrieval condition, with the treated and untreated sections subsequently processed together for immunohistochemistry. Optic densities measured from the same gyrus are normalized to the mean calculated based on the densities obtained from the untreated sections, and plotted in reference to individual sections (totally eight sections). A $\beta$ immunoreactivity in sections preheated at $65^{\circ} \mathrm{C}$ for $1 \mathrm{~h}$ and $3 \mathrm{~h}$ is increased in overall intensity, and specifically in layer I, WM and vascular sites (i.e., cerebral amyloid angiopathy, CAA), relative to untreated (room temperature) control, significantly between the treated and untreated groups but not between the $1 \mathrm{~h}$ and $3 \mathrm{~h}$ groups by Kruskal-Wallis analysis with post hoc test (A-D). On the contrary, sortilin immunoreactivity is not altered in the preheated relative to untreated sections (E-H). Formic acid (100\%) treatment for $1 \mathrm{~h}$ also greatly enhances $\mathrm{A} \beta$ labeling, but has little effect on sortilin labeling (I-M) (paired $t$-test). Guanidine $\mathrm{Hydrochloride} \mathrm{(HCl;}$ $5 \mathrm{M}$ ) pretreatment for $1 \mathrm{~h}$ slightly increases $A \beta$ labeling not reached significance (paired $t$-test), and it has no effect on sortilin labeling related to untreated control (N-R). Cortical layers are marked in the image panels. ${ }^{*} p<0.05$. Scale bar $=100 \mu \mathrm{m}$ in (A) applying to all image panels. 
In this study, we have characterized the normal expression pattern of sortilin in adult human cerebrum with two antibodies targeting separately to the extracellular and intracellular domains. The specificity of the antibodies has been vigorously checked with three approaches, i.e., gene knock-out system, immunogenic peptide absorption and primary antibody omission. Since both antibodies essentially only label the $\sim 100 \mathrm{kDa}$ band in immunoblot of adult/normal rodent and human brain lysates, the immunolabeling seen microscopically would reflect the expression of full-length sortilin in vivo. Based on morphological characteristics, cortical and hippocampal principal neurons express high levels of the full-length protein, particularly in the somatodendritic compartment. The labeled granular elements seen in the somata and dendrites would represent intracellular organelles wherein protein sorting/trafficking takes place (Petersen et al., 1997; Nielsen et al., 2001; Gelling et al., 2012).

Often it is difficult with immunolabeling to determine whether a protein is localized to synaptic structures under the light microscope, especially in human brain samples obtained with postmortem delay. The finding of sortilin immunolabeling to the TE of hilar mossy cells and CA3 pyramidal neurons in normal human brain is remarkable. The visualization of this complex dendritic spine formation by both the extracellular and intracellular domain antibodies strongly suggests that the full-length sortilin protein is actually enriched at postsynaptic sites. Therefore, sortilin could play a physiological role in sorting/recycling of postsynaptic proteins, which could be important for synapse function and plasticity.

\section{Sortilin Fragments Are a Prominent Constituent of Senile Plaques in Human Cerebrum}

Recent studies have explored sortilin in relevance to AD and age-related dementia at genomic, protein and cell biology levels. So far, no data have shown that sortilin gene mutation or polymorphic variation increases the risk of developing $\mathrm{AD}$ (Zeng et al., 2013). Notably, a late study reports that the SNP rs17646665 located in a non-coding region of SORT1 gens is associated with a reduced risk for AD (Andersson et al., 2016). Three studies (to the best of our knowledge) have analyzed the $100 \mathrm{kDa}$ sortilin protein in postmortem human brains, with its levels found either preserved in $\mathrm{AD}$ patients and subjects with mild cognitive impairment relative to people with normal cognition at death (Mufson et al., 2010), or elevated among AD relative to control elderly (Finan et al., 2011; Saadipour et al., 2013). Cell biology and proteomics studies suggest a regulatory interplay between sortilin and the amyloidogenic proteins. Thus, sortilin can mediate APP and BACE1 trafficking (Finan et al., 2011; Saadipour et al., 2013; Yang et al., 2013), while BACE1 and $\gamma$-secretase may catalyze sortilin proteolysis (Nyborg et al., 2006; Hemming et al., 2009).

In the present study, we show that a sortilin C-terminal antibody visualizes extracellularly deposited products in the human brains with cerebral amyloid pathology. The smallest amount of microscopically detectable deposits appears fibrillary, present in the neuropil covering areas about the sizes of neuronal nuclei and somata. The deposits appear to accumulate locally to form mini-plaques, dense-packing and ring-like mature-looking plaques. Neither the isolated deposits nor plaques occur preferentially surrounding the neuronal somata. Also, the deposits are not detectable inside neuronal somata and large dendrites. Quantitative analyses based on fractional areal measurement of individual plaques or collective densitometry of labeled profiles indicate a correlation between sortilin and $A \beta$ deposition in the human cerebrum in a brain region/lamina- and case-dependent manner. Double immunofluorescent characterizations with a panel of markers co-existing at neuritic plaques, i.e., SYN, p-Tau, GFAP and ApoE (Kida et al., 1995; Zhang et al., 2009; Cai et al., 2010, 2012; Sadleir et al., 2016), show a colocalization of sortilin deposits in this type of amyloid plaques.

As elaborated in the "Introduction" Section, senile or neuritic plaques are historically referred to the silver stained lesions consisted of DNs and amorphous material, i.e., the amyloid product recognized later. In $\mathrm{A} \beta$ antibody stain, compact plaques in primitive, dense-packing or cored forms appear to closely represent neuritic plaques (while the so-called "burn-out" plaques may lack or contain only a small amount of DNs; Delaère et al., 1991). A $\beta$ antibodies also label diffuse plaques as well as vascular and meningeal amyloidosis that often co-exist with compact plaques in the brain. Antigen retrieval methods, especially formic acid treatment, are routinely applied to $A \beta$ immunohistochemistry. Diffuse plaques can show fairly dense labeling and mix with compact plaques in the same microscopic field. Thus, microscopic differentiation between the two types may not be always conclusive. In fact, inter-laboratory and interexperimental inconsistency exists in the assessment of plaque lesions (Alafuzoff et al., 2008). Our comparative analyses on the effect of antigen retrieval indicate that section pretreatment with formic acid or heating essentially does not alter the amount and pattern of sortilin deposition, although dramatically enhances $\mathrm{A} \beta$ labeling and affects plaque appearance. Thus, the extracellular sortilin immunolabeling appears to be highly reproducible when used to visualize neuritic-like plaques in postmortem human brain.

Collectively, the data obtained from the human brains through comparative immunohistochemical and immunoblot assessments with the extracellular and intracellular domain antibodies allow us to conclude that the extracellularly deposited sortilin products must be the fragments rather than the parent protein. Specifically, the $15 \mathrm{kDa}$ fragments detected only by the C-terminal antibody appear to be the major culprit behind the extracellular lesions. The levels of these fragments are dramatically elevated in the cortical lysates from AD human brains with amyloid pathology relative to aged/mid-age controls without microscopically overt amyloidosis.

\section{Sortilin Neuropathology Might Relate to Synaptic Degeneration}

As an initially observed human pathology, many mechanistic questions await to address, such as how and why sortilin 


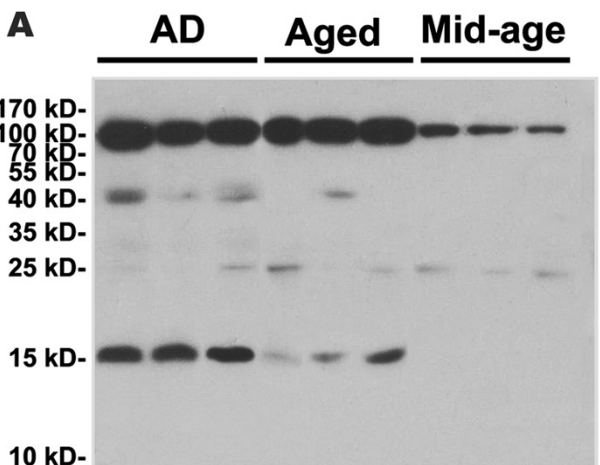

$10 \mathrm{kD}$ -
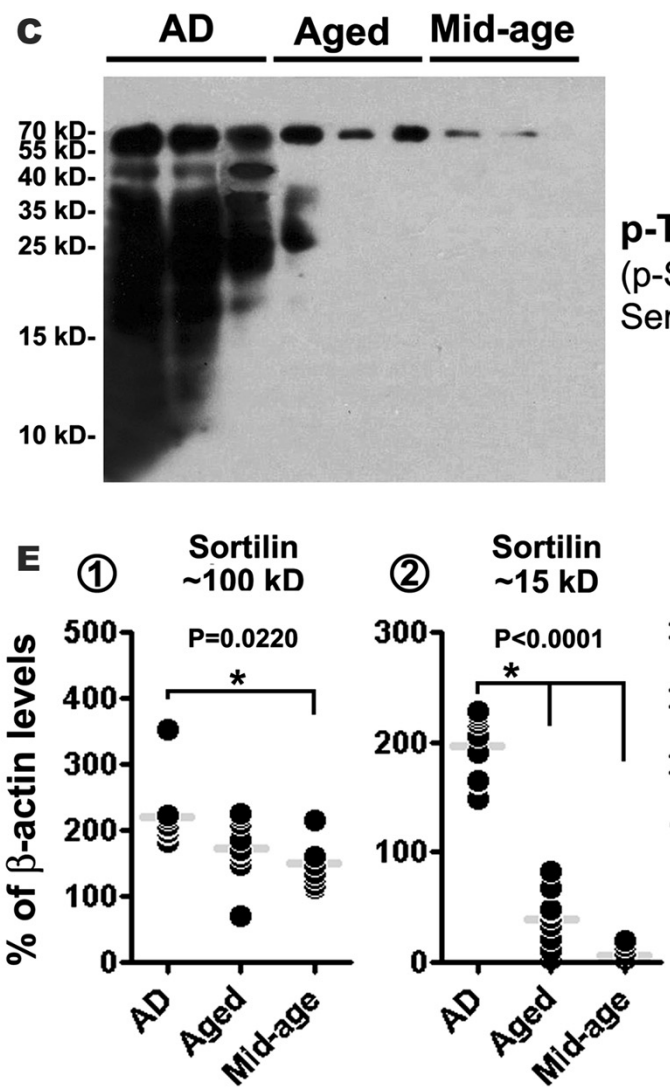

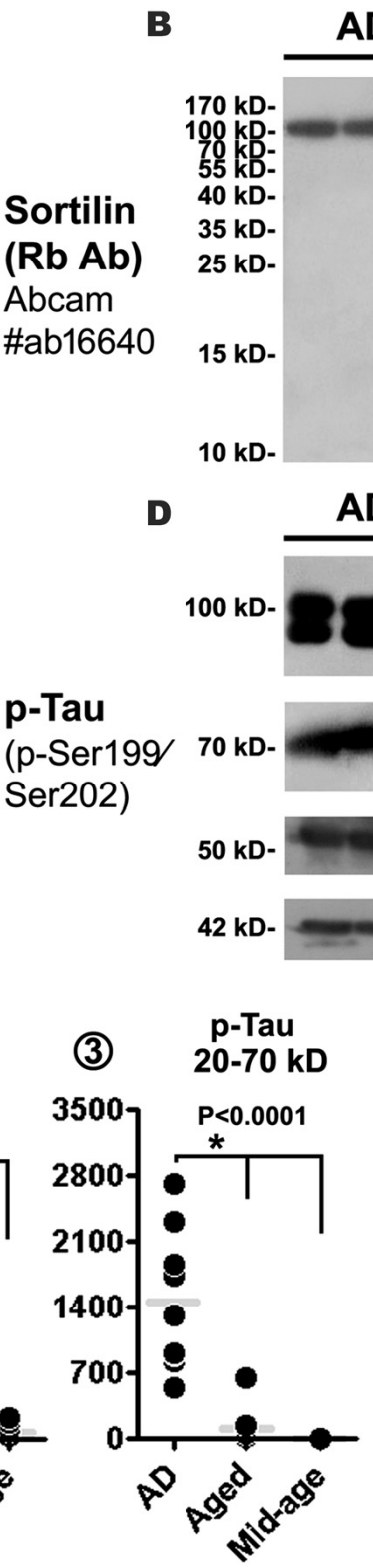

AD Aged Mid-age

\section{Sortilin}

(Gt Ab)

R\&D

Systems

\#AF3154

AD Aged Mid-age

APP

(6E10)

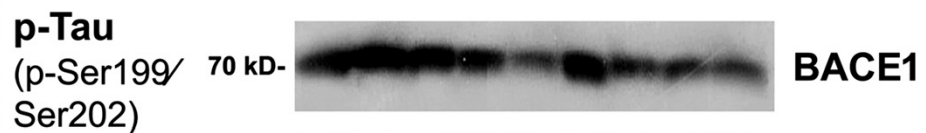
from cases $(n=9)$ with pathologically confirmed amyloid deposition in the contralateral hemisphere, scored $\geq$ B. Samples in the Aged and Mid-age groups are from cases without microscopically evident amyloidosis. Panels (A-D) illustrate a set of representative images (three cases per group) of immunoblots assayed with sortilin and AD related markers, as indicated. Panels (E1-5) show the quantification and statistical result (Kruskal-Wallis non-parametric test with post hoc test, same for below) for each marker based on data from nine cases of each group. The rabbit and goat antibodies detect the same $\sim 100 \mathrm{kDa}$ band representing the full-length sortilin protein, with its median density elevated in the AD relative to Mid-age groups (A,B,E1). The rabbit antibody additionally detects a major fragment at $\sim 15 \mathrm{kDa}$, with its levels elevated in the AD group relative to Aged and Mid-age groups (A,E2). Immunoblotted phosphorylated tau ( $p$-Tau) products appear as a smear of bands less than $70 \mathrm{kDa}$, with the levels measured for 20-70 kDa bands dramatically elevated in the AD relative to Aged and Mid-age groups (C,E3). Levels of amyloid precursor protein (APP) and $\beta$-secretase-1 (BACE1) are also elevated in the AD as compared to the Mid-age group (D,E4,5). Western blot application: 15\% SDS-PAGE gel and $35 \mu \mathrm{g}$ equal protein loading for sortilin and p-Tau antibodies; 10\% SDS-PAGE gel and $35 \mu \mathrm{g}$ equal protein loading for other antibodies. P value for the overall difference between groups is indicated for each marker, with statistically significant inter-group difference $(* P<0.05)$ by post hoc also marked in the graph.

deposition occurs. Our current data allow some preliminary interrogation on a few issues, in reference to existing literature. The first one involves whether sortilin deposition occurs as a direct consequence of amyloidogenesis. While sortilin deposits coexist with $\mathrm{A} \beta$ at neuritic-like plaques in the gray matter, they are not evidently present along with meningeal and 
vascular $\beta$-amyloidosis or in areas with typical diffuse $A \beta$ deposition in the gray and WMs. Accordingly, sortilin deposition could not be coherently explained as a passive event resulted from absorptive binding by $\mathrm{A} \beta$. Neither sortilin full-length nor deposited products are labeled in the DNs. Therefore, it appears unlikely that the sortilin deposits derive from the DNs via secretase-mediated proteolysis of the parent protein. In comparison, increased BACE1 labeling can be found in DNs of neuritic plaques, swollen axons in the WM as well as in vascular and meningeal cells, implicating a potential role of $A \beta$ overproduction by local cellular profiles for site-specific cerebral $\beta$-amyloidosis (Cheng et al., 2014; Deng et al., 2014; Yan et al., 2014; Xue et al., 2015; Devraj et al., 2016).

The second issue is about the anatomic origin of the sortilin deposits. Labeling of the full-length protein is localized primarily to neuronal profiles, enriched in the somatodendritic rather than axonal profiles (as discussed in the first part of "Discussion" Section). The giant spine formations, TE, are distinctly labeled. The labeling is also intense in the ML of the DG and the stratum radiatum of Ammon's horn/subiculum likely in dendritic elements. Thus, dendritic branches and spines could be a major origin of the sortilin deposits. Such a notion fits well with the overall region-specific distribution of sortilin plaques in the neuropil of the gray matter, including the ML and stratum radiatum wherein neuronal somata are rare but plaques are distinct. Synaptic degeneration is considered the most important anatomical basis underlying cognitive decline in $\mathrm{AD}$. Plaque formation appears to be a continuing process tightly associated with synaptic degeneration, with dendritic spine loss and axonal terminal degeneration/dystrophy at the lesion sites (Gonatas et al., 1967; Masliah et al., 1991; Ferrer et al., 1998; Adalbert and Coleman, 2013; Yan et al., 2014; Herms and Dorostkar, 2016; Sadleir et al., 2016). Sortilin fragments might derive largely from degenerating dendritic spines and branches therefore accumulate preferentially at neuritic-like plaques.

The third issue relates to the biochemical process that might underlie the formation of sortilin fragments. Some in vitro and in vivo data suggest that the extracellular domain of sortilin can shed off as soluble products (Navarro et al., 2002; Molgaard et al., 2016; Ogawa et al., 2016). The $\sim 15 \mathrm{kDa}$ products are exclusively immunoblotted by the C-terminal antibody, with the plaque labeling completely diminished by the corresponding blocking immunogenic peptide. With this information and in reference to the molecular weight $(\sim 15 \mathrm{kDa})$, the deposited sortilin products could be predicted to contain the C-terminal tail, transmembrane domain and a short part of the extracellular sequence (roughly a.a. 670-831 according to online protein analysis tools), without considering the situation of protein modification (the C-terminal

\section{REFERENCES}

Adalbert, R., and Coleman, M. P. (2013). Review: axon pathology in age-related neurodegenerative disorders. Neuropathol. Appl. Neurobiol. 39, 90-108. doi: 10.1111/j.1365-2990.2012.01308.x

Aisen, P. S. (2005). The development of anti-amyloid therapy for Alzheimer's disease: from secretase modulators to polymerisation inhibitors. CNS Drugs 19, 989-996. doi: 10.2165/00023210-200519120-00002 region contains two putative phosphorylation sites). The sortilin fragments might be formed after the extracellular domain is cleaved off, and aberrantly accumulate site-specifically along with dendritic/spine degeneration of local neurons during plaque pathogenesis.

In summary, we have identified sortilin fragments as a new component of the abnormally deposited protein products in senile plaques in the human cerebrum. This sortilin extracellular pathology is fairly preferentially associated with neuritic-like amyloid plaques, but rarely occurs around the pia, at vascular wall and in regions with typical diffuse $A \beta$ deposition. The plaque-like sortilin labeling is not affected by antigen retrieval methods that enhance $\mathrm{A} \beta$ immunoreactivity. The $\sim 15 \mathrm{kDa}$ fragments appear to be a major form of the deposited components, which is predicted to derive largely from the C-terminal region likely including the transmembrane part of the parent protein. Mechanistically, we speculate that these fragments are formed along with the degeneration of largely the dendritic spines and fine branches.

\section{AUTHOR CONTRIBUTIONS}

$\mathrm{XH}$ : immunohistochemistry and immunofluorescence; ZLH and C-QL: western blot; ZL: confocal imaging; C-SR: antibody verification; W-YQ and CM: human brain banking and pathological characterization; AP, LS and B-ST: brain banking; YCai: imaging and data analysis; YChu: verification of the pathology in Caucasian AD brain samples; HC: data interpretation and article writing; X-FZ: antibody verification and data interpretation; X-XY: experimental design, human brain banking and pathological characterization, data analysis and interpretation; article writing.

\section{ACKNOWLEDGMENTS}

This study was supported by National Natural Science Foundation of China (\#31371095 and \#91632116, X-XY), an intramural program of the National Institute on Aging (HC) and Australian National Health and Medical Research Council (APP1021408, X-FZ).

\section{SUPPLEMENTARY MATERIAL}

The Supplementary Material for this article can be found online at: http://journal.frontiersin.org/article/10.3389/fnana.2017. 00045/full\#supplementary-material

Alafuzoff, I., Pikkarainen, M., Arzberger, T., Thal, D. R., Al-Sarraj, S., Bell, J., et al. (2008). Inter-laboratory comparison of neuropathological assessments of beta-amyloid protein: a study of the brainnet europe consortium. Acta Neuropathol. 115, 533-546. doi: 10.1007/s00401-0080358-2

Allsop, D., Landon, M., Kidd, M., Lowe, J. S., Reynolds, G. P., and Gardner, A. (1986). Monoclonal antibodies raised against a subsequence of senile plaque core protein react with plaque cores, plaque periphery and cerebrovascular 
amyloid in Alzheimer's disease. Neurosci. Lett. 68, 252-256. doi: 10.1016/03043940(86)90152-7

Andersson, C. H., Hansson, O., Minthon, L., Andreasen, N., Blennow, K., Zetterberg, H., et al. (2016). A genetic variant of the sortilin 1 gene is associated with reduced risk of Alzheimer's disease. J. Alzheimers Dis. 53, 1353-1363. doi: $10.3233 /$ jad-160319

Andreasen, N., Minthon, L., Davidsson, P., Vanmechelen, E., Vanderstichele, H., Winblad, B., et al. (2001). Evaluation of CSF-tau and CSF-A 342 as diagnostic markers for Alzheimer disease in clinical practice. Arch. Neurol. 58, 373-379. doi: 10.1001/archneur.58.3.373

Avci, D., and Lemberg, M. K. (2015). Clipping or extracting: two ways to membrane protein degradation. Trends Cell Biol. 25, 611-622. doi: 10.1016/j. tcb.2015.07.003

Borchelt, D. R., Ratovitski, T., van Lare, J., Lee, M. K., Gonzales, V., Jenkins, N. A., et al. (1997). Accelerated amyloid deposition in the brains of transgenic mice coexpressing mutant presenilin 1 and amyloid precursor proteins. Neuron 19, 939-945. doi: 10.1016/s0896-6273(00) 80974-5

Braak, H., Alafuzoff, I., Arzberger, T., Kretzschmar, H., and Del Tredici, K. (2006). Staging of Alzheimer disease-associated neurofibrillary pathology using paraffin sections and immunocytochemistry. Acta Neuropathol. 112, 389-404. doi: 10.1007/s00401-006-0127-z

Braak, H., and Braak, E. (1991). Demonstration of amyloid deposits and neurofibrillary changes in whole brain sections. Brain Pathol. 1, 213-216. doi: 10.1111/j.1750-3639.1991.tb00661.x

Burns, M. P., Noble, W. J., Olm, V., Gaynor, K., Casey, E., LaFrancois, J., et al. (2003). Co-localization of cholesterol, apolipoprotein E and fibrillar $\mathrm{A} \beta$ in amyloid plaques. Mol. Brain Res. 110, 119-125. doi: 10.1016/s0169328x(02)00647-2

Cai, Y., Xiong, K., Zhang, X. M., Cai, H., Luo, X. G., Feng, J. C., et al. (2010). $\beta$-secretase-1 elevation in aged monkey and Alzheimer's disease human cerebral cortex occurs around the vasculature in partnership with multisystem axon terminal pathogenesis and $\beta$-amyloid accumulation. Eur. J. Neurosci. 32, 1223-1238. doi: 10.1111/j.1460-9568.2010. 07376.x

Cai, Y., Zhang, X. M., Macklin, L. N., Cai, H., Luo, X. G., Oddo, S., et al. (2012). BACE1 elevation is involved in amyloid plaque development in the triple transgenic model of Alzheimer's disease: differential A $\beta$ antibody labeling of early-onset axon terminal pathology. Neurotox. Res. 21, 160-174. doi: 10.1007/s12640-011-9256-9

Carlo, A. S. (2013). Sortilin, a novel APOE receptor implicated in Alzheimer disease. Prion 7, 378-382. doi: 10.4161/pri.26746

Chen, Z. Y., Ieraci, A., Teng, H., Dall, H., Meng, C. X., Herrera, D. G., et al. (2005). Sortilin controls intracellular sorting of brain-derived neurotrophic factor to the regulated secretory pathway. J. Neurosci. 25, 6156-6166. doi: 10.1523/JNEUROSCI.1017-05.2005

Cheng, X., He, P., Yao, H., Dong, Q., Li, R., and Shen, Y. (2014). Occludin deficiency with BACE1 elevation in cerebral amyloid angiopathy. Neurology 82, 1707-1715. doi: 10.1212/WNL.0000000000000403

Coria, F., Castaño, E., Prelli, F., Larrondo-Lillo, M., van Duinen, S., Shelanski, M. L., et al. (1988). Isolation and characterization of amyloid P component from Alzheimer's disease and other types of cerebral amyloidosis. Lab. Invest. 58, 454-458.

Coutinho, M. F., Bourbon, M., Prata, M. J., and Alves, S. (2013). Sortilin and the risk of cardiovascular disease. Rev. Port. Cardiol. 32, 793-799. doi: 10.1016/j. repc.2013.02.006

Cristóvão, J. S., Santos, R., and Gomes, C. M. (2016). Metals and neuronal metal binding proteins implicated in Alzheimer's disease. Oxid. Med. Cell. Longev. 2016:9812178. doi: 10.1155/2016/9812178

Critchley, M. (1929). Critical review: the nature and significance of senile plaques. J. Neurol. Psychopathol. 10, 124-139. doi: 10.1136/jnnp.s1-10. 38.124

Delaère, P., Duyckaerts, C., He, Y., Piette, F., and Hauw, J. J. (1991). Subtypes and differential laminar distributions of A $\beta 4$ deposits in Alzheimer's disease: relationship with the intellectual status of 26 cases. Acta Neuropathol. 81, 328-335. doi: 10.1007/bf00305876

Deng, X., Li, M., Ai, W., He, L., Lu, D., Patrylo, P. R., et al. (2014). Lipolysaccharide-induced neuroinflammation is associated with
Alzheimer-like amyloidogenic axonal pathology and dendritic degeneration in rats. Adv. Alzheimer Dis. 3, 78-93. doi: 10.4236/aad.2014.32009

Devraj, K., Poznanovic, S., Spahn, C., Schwall, G., Harter, P. N., Mittelbronn, M., et al. (2016). BACE-1 is expressed in the blood-brain barrier endothelium and is upregulated in a murine model of Alzheimer's disease. J. Cereb. Blood Flow Metab. 36, 1281-1294. doi: 10.1177/0271678X15606463

Eriksson, S., Janciauskiene, S., and Lannfelt, L. (1995). Alpha 1-antichymo-trypsin regulates Alzheimer beta-amyloid peptide fibril formation. Proc. Natl. Acad. Sci. US A 92, 2313-2317. doi: 10.1073/pnas.92.6.2313

Felsky, D., Szeszko, P., Yu, L., Honer, W. G., De Jager, P. L., Schneider, J. A., et al. (2014). The SORL1 gene and convergent neural risk for Alzheimer's disease across the human lifespan. Mol. Psychiatry 19, 1125-1132. doi: 10.1038/mp. 2013.142

Ferrer, I., Martí, E., Tortosa, A., and Blasi, J. (1998). Dystrophic neurites of senile plaques are defective in proteins involved in exocytosis and neurotransmission. J. Neuropathol. Exp. Neurol. 57, 218-225. doi: 10.1097/00005072-19980300000002

Finan, G. M., Okada, H., and Kim, T. W. (2011). BACE1 retrograde trafficking is uniquely regulated by the cytoplasmic domain of sortilin. J. Biol. Chem. 286, 12602-12616. doi: 10.1074/jbc.M110.170217

Garai, K., Verghese, P. B., Baban, B., Holtzman, D. M., and Frieden, C. (2014). The binding of apolipoprotein $\mathrm{E}$ to oligomers and fibrils of amyloid$\beta$ alters the kinetics of amyloid aggregation. Biochemistry 53, 6323-6331. doi: $10.1021 /$ bi5008172

García-Marín, V., García-López, P., and Freire, M. (2007). Cajal's contributions to the study of Alzheimer's disease. J. Alzheimers Dis. 12, 161-174. doi: $10.3233 /$ jad-2007-12206

Gelling, C. L., Dawes, I. W., Perlmutter, D. H., Fisher, E. A., and Brodsky, J. L. (2012). The endosomal protein-sorting receptor sortilin has a role in trafficking $\alpha-1$ antitrypsin. Genetics 192, 889-903. doi: 10.1534/genetics.112.143487

Glenner, G. G., and Wong, C. W. (1984). Alzheimer's disease: initial report of the purification and characterization of a novel cerebrovascular amyloid protein. Biochem. Biophys. Res. Commun. 120, 885-890. doi: 10.1016/s0006$291 x(84) 80190-4$

Gonatas, N. K., Anderson, W., and Evangelista, I. (1967). The contribution of altered synapses in the senile plaque: an electron microscopic study in Alzheimer's dementia. J. Neuropathol. Exp. Neurol. 26, 25-39. doi: 10.1097/00005072-196701000-00003

Griffith, C. M., Xie, M. X., Qiu, W. Y., Sharp, A. A., Ma, C., Pan, A., et al. (2016). Aberrant expression of the pore-forming $\mathrm{K}_{A T P}$ channel subunit Kir6.2 in hippocampal reactive astrocytes in the $3 \times \mathrm{Tg}-\mathrm{AD}$ mouse model and human Alzheimer's disease. Neuroscience 336, 81-101. doi: 10.1016/j.neuroscience. 2016.08.034

Hemming, M. L., Elias, J. E., Gygi, S. P., and Selkoe, D. J. (2009). Identification of beta-secretase (BACE1) substrates using quantitative proteomics. PLoS One 4:e8477. doi: 10.1371/journal.pone.0008477

Herholz, K., and Ebmeier, K. (2011). Clinical amyloid imaging in Alzheimer's disease. Lancet Neurol. 10, 667-670. doi: 10.1016/S1474-4422(11)70123-5

Hermey, G. (2009). The Vps10p-domain receptor family. Cell. Mol. Life Sci. 66, 2677-2689. doi: 10.1007/s00018-009-0043-1

Herms, J., and Dorostkar, M. M. (2016). Dendritic spine pathology in neurodegenerative diseases. Annu. Rev. Pathol. 11, 221-250. doi: 10.1146/annurev-pathol-012615-044216

Hsiao, K., Chapman, P., Nilsen, S., Eckman, C., Harigaya, Y., Younkin, S., et al. (1996). Correlative memory deficits, $A \beta$ elevation and amyloid plaques in transgenic mice. Science 274, 99-102. doi: 10.1126/science.274.5284.99

Hu, F., Padukkavidana, T., Vægter, C. B., Brady, O. A., Zheng, Y., Mackenzie, I. R., et al. (2010). Sortilin-mediated endocytosis determines levels of the frontotemporal dementia protein, progranulin. Neuron 68, 654-667. doi: 10.1016/j.neuron.2010.09.034

Jellinger, K. A., and Bancher, C. (1988). Neuropathology of Alzheimer's disease: a critical update. J. Neural. Transm. Suppl. 54, 77-95. doi: 10.1007/978-3-70917508-8_8

Karran, E., and De Strooper, B. (2016). The amyloid cascade hypothesis: are we poised for success or failure? J. Neurochem. 139, 237-252. doi: 10.1111/jnc. 13632

Kida, E., Choi-Miura, N. H., and Wisniewski, K. E. (1995). Deposition of apolipoproteins $\mathrm{E}$ and $\mathrm{J}$ in senile plaques is topographically determined in 
both Alzheimer's disease and down's syndrome brain. Brain Res. 685, 211-216. doi: 10.1016/0006-8993(95)00482-6

Kjolby, M., Nielsen, M. S., and Petersen, C. M. (2015). Sortilin, encoded by the cardiovascular risk gene SORT1 and its suggested functions in cardiovascular disease. Curr. Atheroscler. Rep. 17:496. doi: 10.1007/s11883-0150496-7

Louwersheimer, E., Ramirez, A., Cruchaga, C., Becker, T., Kornhuber, J., Peters, O., et al. (2015). Influence of genetic variants in SORL1 gene on the manifestation of Alzheimer's disease. Neurobiol. Aging 36, 1605.e13-1605.20. doi: 10.1016/j.neurobiolaging.2014.12.007

Luse, S. A., and Smith, K. R. Jr. (1964). The ultrastructure of senile plaques. Am. J. Pathol. 44, 553-563.

Masliah, E., Hansen, L., Albright, T., Mallory, M., and Terry, R. D. (1991). Immunoelectron microscopic study of synaptic pathology in Alzheimer's disease. Acta Neuropathol. 81, 428-433. doi: 10.1007/bf00293464

Masters, C. L., Simms, G., Weinman, N. A., Multhaup, G., McDonald, B. L., and Beyreuther, K. (1985). Amyloid plaque core protein in Alzheimer disease and down syndrome. Proc. Natl. Acad. Sci. U S A 82, 4245-4249. doi: 10.1073/pnas. 82.12 .4245

Mathis, C. A., Bacskai, B. J., Kajdasz, S. T., McLellan, M. E., Frosch, M. P., Hyman, B. T., et al. (2002). A lipophilic thioflavin-T derivative for positron emission tomography (PET) imaging of amyloid in brain. Bioorg. Med. Chem. Lett. 12, 295-298. doi: 10.1016/S0960-894X(01)00734-X

Mazella, J., Zsürger, N., Navarro, V., Chabry, J., Kaghad, M., Caput, D., et al. (1998). The $100-\mathrm{kDa}$ neurotensin receptor is gp95/sortilin, a non-G-proteincoupled receptor. J. Biol. Chem. 273, 26273-26276. doi: 10.1074/jbc.273.41. 26273

Molgaard, S., Demontis, D., Nicholson, A. M., Finch, N. A., Petersen, R. C., Petersen, C. M., et al. (2016). Soluble sortilin is present in excess and positively correlates with progranulin in CSF of aging individuals. Exp. Gerontol. 84, 96-100. doi: 10.1016/j.exger.2016.09.002

Montine, T. J., Phelps, C. H., Beach, T. G., Bigio, E. H., Cairns, N. J., Dickson, D. W., et al. (2012). National institute on aging-Alzheimer's association guidelines for the neuropathologic assessment of Alzheimer's disease: a practical approach. Acta Neuropathol. 123, 1-11. doi: 10.1007/s00401-011-0910-3

Mortensen, M. B., Kjolby, M., Gunnersen, S., Larsen, J. V., Palmfeldt, J., Falk, E., et al. (2014). Targeting sortilin in immune cells reduces proinflammatory cytokines and atherosclerosis. J. Clin. Invest. 124, 5317-5322. doi: 10.1172/JCI76002

Mufson, E. J., Wuu, J., Counts, S. E., and Nykjaer, A. (2010). Preservation of cortical sortilin protein levels in MCI and Alzheimer's disease. Neurosci. Lett. 471, 129-133. doi: 10.1016/j.neulet.2010.01.023

Navarro, V., Vincent, J. P., and Mazella, J. (2002). Shedding of the luminal domain of the neurotensin receptor-3/sortilin in the HT29 cell line. Biochem. Biophys. Res. Commun. 29, 760-764. doi: 10.1016/s0006-291x(02)0 2564-0

Nielsen, M. S., Madsen, P., Christensen, E. I., Nykjaer, A., Gliemann, J., Kasper, D., et al. (2001). The sortilin cytoplasmic tail conveys golgi-endosome transport and binds the VHS domain of the GGA2 sorting protein. EMBO J. 20, 2180-2190. doi: 10.1093/emboj/20.9.2180

Nyborg, A. C., Ladd, T. B., Zwizinski, C. W., Lah, J. J., and Golde, T. E. (2006). Sortilin, SorCS1b and SorLA Vps10p sorting receptors, are novel gammasecretase substrates. Mol. Neurodegener. 1:3. doi: 10.1186/1750-1326-1-3

Nykjaer, A., Lee, R., Teng, K. K., Jansen, P., Madsen, P., Nielsen, M. S., et al. (2004). Sortilin is essential for proNGF-induced neuronal cell death. Nature 427, 843-848. doi: 10.1038/nature02319

Oakley, H., Cole, S. L., Logan, S., Maus, E., Shao, P., Craft, J., et al. (2006). Intraneuronal $\beta$-amyloid aggregates, neurodegeneration and neuron loss in transgenic mice with 5 familial Alzheimer's disease mutations: potential factors in amyloid plaque formation. J. Neurosci. 26, 10129-10140. doi: 10.1523/JNEUROSCI.1202-06.2006

Oddo, S., Caccamo, A., Shepherd, J. D., Murphy, M. P., Golde, T. E., Kayed, R., et al. (2003). Triple-transgenic model of Alzheimer's disease with plaques and tangles: intracellular A $\beta$ and synaptic dysfunction. Neuron 39, 409-421. doi: 10.1016/S0896-6273(03)00434-3

Ogawa, K., Ueno, T., Iwasaki, T., Kujiraoka, T., Ishihara, M., Kunimoto, S., et al. (2016). Soluble sortilin is released by activated platelets and its circulating levels are associated with cardiovascular risk factors. Atherosclerosis 249, 110-115. doi: 10.1016/j.atherosclerosis.2016.03.041

Ohry, A., and Buda, O. (2015). Teofil Simchowicz (1879-1957): the scientist who coined senile plaques in neuropathology. Rom. J. Morphol. Embryol. 56, 1545-1548. Available online at: http://www.rjme. ro/RJME/resources/files/56041515451548.pdf

Oifa, A. I. (1973). Paul Divry-founder of the concept of cerebral amyloidosis. $Z h$. Nevropatol. Psikhiatr. Im. S S Korsakova 73, 1078-1082.

Petersen, C. M., Nielsen, M. S., Nykjaer, A., Jacobsen, L., Tommerup, N., Rasmussen, H. H., et al. (1997). Molecular identification of a novel candidate sorting receptor purified from human brain by receptor-associated protein affinity chromatography. J. Biol. Chem. 272, 3599-3605. doi: 10.1074/jbc.272. 6.3599

Poo, M. M., Du, J. L., Ip, N. Y., Xiong, Z. Q., Xu, B., and Tan, T. (2016). China brain project: basic neuroscience, brain diseases and braininspired computing. Neuron 92, 591-596. doi: 10.1016/j.neuron.2016. 10.050

Pottier, C., Hannequin, D., Coutant, S., Rovelet-Lecrux, A., Wallon, D. Rousseau, S., et al. (2012). High frequency of potentially pathogenic SORL1 mutations in autosomal dominant early-onset alzheimer disease. Mol. Psychiatry 17, 875-879. doi: 10.1038/mp.2012.15

Reitz, C., Tokuhiro, S., Clark, L. N., Conrad, C., Vonsattel, J. P., Hazrati, L. N., et al. (2011). SORCS1 alters amyloid precursor protein processing and variants may increase Alzheimer's disease risk. Ann. Neurol. 69, 47-64. doi: 10.1002/ana. 22308

Robakis, N. K., Wisniewski, H. M., Jenkins, E. C., Devine-Gage, E. A., Houck, G. E., Yao, X. L., et al. (1987). Chromosome 21q21 sublocalization of gene encoding $\beta$-amyloid peptide in cerebral vessels and neuritic (senile) plaques of people with Alzheimer disease and down syndrome. Lancet 1, 384-385.

Rogaeva, E., Meng, Y., Lee, J. H., Gu, Y., Kawarai, T., Zou, F., et al. (2007). The neuronal sortilin-related receptor SORL1 is genetically associated with Alzheimer disease. Nat. Genet. 39, 168-177. doi: 10.1038/ng 1943

Rogers, J., Cooper, N. R., Webster, S., Schultz, J., McGeer, P. L., Styren, S. D., et al. (1992). Complement activation by $\beta$-amyloid in Alzheimer disease. Proc. Natl. Acad. Sci. U S A 89, 10016-10020.

Ruan, C. S., Yang, C. R., Li, J. Y., Luo, H. Y., Bobrovskaya, L., and Zhou, X. F. (2016). Mice with sortl deficiency display normal cognition but elevated anxiety-like behavior. Exp. Neurol. 281, 99-108. doi: 10.1016/j.expneurol.2016. 04.015

Saadipour, K., Yang, M., Lim, Y., Georgiou, K., Sun, Y., Keating, D., et al. (2013). Amyloid beta $(A \beta)$ up-regulates the expression of sortilin via the p75 (NTR)/RhoA signaling pathway. J. Neurochem. 127, 152-162. doi: 10.1111/jnc. 12383

Sadleir, K. R., Kandalepas, P. C., Buggia-Prévot, V., Nicholson, D. A., Thinakaran, G., and Vassar, R. (2016). Presynaptic dystrophic neurites surrounding amyloid plaques are sites of microtubule disruption, BACE1 elevation and increased $A \beta$ generation in Alzheimer's disease. Acta Neuropathol. 132, 235-256. doi: 10.1007/s00401-0161558-9

Sarret, P., Krzywkowski, P., Segal, L., Nielsen, M. S., Petersen, C. M., Mazella, J., et al. (2003). Distribution of NTS3 receptor/sortilin mRNA and protein in the rat central nervous system. J. Comp. Neurol. 461, 483-505. doi: 10.1002/cne. 10708

Schwarzman, A. L., Gregori, L., Vitek, M. P., Lyubski, S., Strittmatter, W. J., Enghilde, J. J., et al. (1994). Transthyretin sequesters amyloid beta protein and prevents amyloid formation. Proc. Natl. Acad. Sci. U S A 91, 8368-8372. doi: 10.1016/0197-4580(94)92672-7

Shea, Y. F., Chu, L. W., Chan, A. O., Ha, J., Li, Y., and Song, Y. Q. (2016). A systematic review of familial Alzheimer's disease: differences in presentation of clinical features among 3 mutated genes and potential ethnic differences. J. Formos. Med. Assoc. 115, 67-75. doi: 10.1016/j.jfma.2015. 08.004

Strong, A., Ding, Q., Edmondson, A. C., Millar, J. S., Sachs, K. V., $\mathrm{Li}, \mathrm{X}$., et al. (2012). Hepatic sortilin regulates both apolipoprotein B secretion and LDL catabolism. J. Clin. Invest. 122, 2807-2816. doi: 10.1172/ JCI63563 
Struble, R. G., Cork, L. C., Whitehouse, P. J., and Price, D. L. (1982). Cholinergic innervation in neuritic plaques. Science 216, 413-415. doi: 10.1126/science. 6803359

Struble, R. G., Powers, R. E., Casanova, M. F., Kitt, C. A., Brown, E. C., and Price, D. L. (1987). Neuropeptidergic systems in plaques of Alzheimer's disease. J. Neuropathol. Exp. Neurol. 46, 567-584.

Vassar, R., Kovacs, D. M., Yan, R., and Wong, P. C. (2009). The $\beta$-secretase enzyme BACE in health and Alzheimer's disease: regulation, cell biology, function and therapeutic potential. J. Neurosci. 29, 12787-12794. doi: 10.1523/JNEUROSCI. 3657-09.2009

Verheijen, J., Van den Bossche, T., van der Zee, J., Engelborghs, S., SanchezValle, R., Lladó, A., et al. (2016). A comprehensive study of the genetic impact of rare variants in SORL1 in European early-onset Alzheimer's disease. Acta Neuropathol. 132, 213-224. doi: 10.1007/s00401-016-1566-9

Walker, L. C., Kitt, C. A., Struble, R. G., Schmechel, D. E., Oertel, W. H., Cork, L. C., et al. (1985). Glutamic acid decarboxylase-like immunoreactive neurites in senile plaques. Neurosci. Lett. 59, 165-169. doi: 10.1016/03043940(85)90194-6

Watson, M. D., Roher, A. E., Kim, K. S., Spiegel, K., and Emmerling, M. (1997). Complement interactions with amyloid $\beta 1-42$ : a nidus for inflammation in $\mathrm{AD}$ brains. Amyloid 4, 147-156. doi: 10.3109/13506129709014379

Wen, Y., Miyashita, A., Kitamura, N., Tsukie, T., Saito, Y., Hatsuta, H., et al. (2013). SORL1 is genetically associated with neuropathologically characterized late-onset Alzheimer's disease. J. Alzheimers Dis. 35, 387-394. doi: 10.3233/JAD-122395

Westergaard, U. B., Sørensen, E. S., Hermey, G., Nielsen, M. S., Nykjaer, A., Kirkegaard, K., et al. (2004). Functional organization of the sortilin Vps10p domain. J. Biol. Chem. 279, 50221-50229. doi: 10.1074/jbc. M408873200

Wolfe, M. S., and Haass, C. (2001). The role of presenilins in gammasecretase activity. J. Biol. Chem. 276, 5413-5416. doi: 10.1074/jbc. R000026200

Wu, C. W., Liao, P. C., Yu, L., Wang, S. T., Chen, S. T., Wu, M., et al. (2004). Hemoglobin promotes $A \beta$ oligomer formation and localizes in neurons and amyloid deposits. Neurobiol. Dis. 17, 367-377. doi: 10.1016/j.nbd.2004. 08.014

Xue, Z. Q., He, Z. W., Yu, J. J., Cai, Y., Qiu, W. Y., Pan, A., et al. (2015). Nonneuronal and neuronal BACE1 elevation in association with angiopathic and leptomeningeal $\beta$-amyloid deposition in the human brain. BMC Neurol. 15:71. doi: 10.1186/s12883-015-0327-Z

Yamaguchi, H., Hirai, S., Morimatsu, M., Shoji, M., and Ihara, Y. (1988). A variety of cerebral amyloid deposits in the brains of the Alzheimer-type dementia demonstrated by beta protein immunostaining. Acta Neuropathol. 76, 541-549. doi: 10.1007/bf00689591

Yan, X. X., Ma, C., Gai, W. P., Cai, H., and Luo, X. G. (2014). Can BACE1 inhibition mitigate early axonal pathology in neurological diseases? J. Alzheimers Dis. 38, 705-718. doi: 10.3233/JAD-131400

Yan, X. X., Ma, C., Bao, A. M., Wang, X. M., and Gai, W. P. (2015). Brain banking as a cornerstone of neuroscience in China. Lancet Neurol. 14:136. doi: 10.1016/s1474-4422(14)70259-5

Yang, M., Lim, Y., Li, X., Zhong, J. H., and Zhou, X. F. (2011). Precursor of brainderived neurotrophic factor (proBDNF) forms a complex with huntingtinassociated protein-1 (HAP1) and sortilin that modulates proBDNF trafficking, degradation and processing. J. Biol. Chem. 286, 16272-16284. doi: 10.1074/jbc. M110.195347

Yang, M., Virassamy, B., Vijayaraj, S. L., Lim, Y., Saadipour, K., Wang, Y. J., et al. (2013). The intracellular domain of sortilin interacts with amyloid precursor protein and regulates its lysosomal and lipid raft trafficking. PLoS One 8:e63049. doi: 10.1371/journal.pone.0063049

Zeng, F., Deng, Y. P., Yi, X., Cao, H. Y., Zou, H. Q., Wang, X., et al. (2013). No association of SORT1 gene polymorphism with sporadic Alzheimer's disease in the chinese han population. Neuroreport 24, 464-468. doi: 10.1097/WNR. ob013e3283619f43

Zhang, X. M., Cai, Y., Xiong, K., Cai, H., Luo, X. G., Feng, J. C., et al. (2009). Beta-secretase-1 elevation in transgenic mouse models of Alzheimer's disease is associated with synaptic/axonal pathology and amyloidogenesis: implications for neuritic plaque development. Eur. J. Neurosci. 30, 2271-2283. doi: 10.1111/j. 1460-9568.2009.07017.x

Zhu, H. X., Xue, Z. Q., Qiu, W. Y., Zeng, Z. J., Dai, J. P., Ma, C., et al. (2015). Age-related intraneuronal accumulation of $\alpha$ II-spectrin breakdown product SBDP120 in the human cerebrum is enhanced in Alzheimer's disease. Exp. Gerontol. 69, 43-52. doi: 10.1016/j.exger.2015.06.003

Conflict of Interest Statement: The authors declare that the research was conducted in the absence of any commercial or financial relationships that could be construed as a potential conflict of interest.

Copyright (C) $2017 \mathrm{Hu}, \mathrm{Hu}, \mathrm{Li}$, Ruan, Qiu, Pan, Li, Cai, Shen, Chu, Tang, Cai, Zhou, Ma and Yan. This is an open-access article distributed under the terms of the Creative Commons Attribution License (CC BY). The use, distribution or reproduction in other forums is permitted, provided the original author(s) or licensor are credited and that the original publication in this journal is cited, in accordance with accepted academic practice. No use, distribution or reproduction is permitted which does not comply with these terms. 